\title{
Application of Approximate Unsteady Aerodynamics for Flutter Analysis
}

\author{
Chan-gi Pak ${ }^{*}$ and Wesley W. $\mathrm{Li}^{\dagger}$ \\ NASA Dryden Flight Research Center, Edwards, CA 93523-0273
}

\begin{abstract}
A technique for approximating the modal aerodynamic influence coefficient (AIC) matrices by using basis functions has been developed. A process for using the resulting approximated modal AIC matrix in aeroelastic analysis has also been developed. The method requires the unsteady aerodynamics in frequency domain, and this methodology can be applied to the unsteady subsonic, transonic, and supersonic aerodynamics. The flutter solution can be found by the classic methods, such as rational function approximation, $k$, $p-k$, $p$, root locus et cetera. The unsteady aeroelastic analysis using unsteady subsonic aerodynamic approximation is demonstrated herein. The technique presented is shown to offer consistent flutter speed prediction on an aerostructures test wing (ATW) 2 and a hybrid wing body (HWB) type of vehicle configuration with negligible loss in precision. This method computes AICs that are functions of the changing parameters being studied and are generated within minutes of CPU time instead of hours. These results may have practical application in parametric flutter analyses as well as more efficient multidisciplinary design and optimization studies.
\end{abstract}

\section{Nomenclature}

$\begin{array}{lll}A & = & \text { general AIC matrix } \\ \text { AIC } & = & \text { aerodynamic influence coefficient } \\ \text { ATW } & = & \text { aerostructures test wing } \\ \text { BFA } & = & \text { basis function approximation } \\ \text { CEM } & = & \text { central executive module } \\ \text { DFRC } & = & \text { Dryden Flight Research Center } \\ \mathrm{g} & = & \text { damping } \\ \text { HWB } & = & \text { hybrid wing body } \\ k & = & \text { reduced frequency } \\ \text { MDAO } & = & \text { multidisciplinary design, analysis, and optimization } \\ m & = & \text { number of mode shapes } \\ \text { NASA } & = & \text { National Aeronautics and Space Administration } \\ n & = & \text { number of basis functions } \\ Q & = & \text { modal AIC matrix } \\ Q_{i j} & = & \text { i-th row and j-th column element of the modal AIC matrix } Q \\ \bar{Q} & = & \text { approximate modal AIC matrix } \\ \bar{Q}_{i j} & = & \text { i-th row and j-th column element of the approximate modal AIC matrix } \bar{Q} \\ \tilde{Q} & = & \text { basis AIC matrix } \\ \tilde{Q} & = & \text { velocity row and r-th column element of the basis AIC matrix } \tilde{Q} \\ \mathrm{~V} & =\end{array}$

\footnotetext{
* Leader, Structural Dynamics Group, Aerostructures Branch, P.O. Box 273/MS 48201A, Member AIAA.

$\dagger$ Structural Dynamics Group, Aerostructures Branch, P.O. Box 273/MS 48202A, Member AIAA.
} 


$\begin{array}{lll}\beta_{k}^{i} & = & \text { Modal participation factors of the } \mathrm{k} \text {-th basis function on the } \mathrm{i} \text {-th mode shape } \\ \beta_{S}^{i} & = & \text { Modal participation factors of the } \mathrm{s}-\mathrm{th} \text { basis function on the } \mathrm{i} \text {-th mode shape } \\ \beta_{k}^{j} & = & \text { Modal participation factors of the } \mathrm{k} \text {-th basis function on the } \mathrm{j} \text {-th mode shape } \\ \beta_{r}^{j} & = & \text { Modal Participation factors of the } \mathrm{r} \text {-th basis function on the } \mathrm{j} \text {-th mode shape } \\ \Phi & = & \text { modal matrix } \\ \phi & = & \text { mode shape } \\ \phi_{\mathrm{i}} & = & \text { i-th mode shape } \\ \Psi_{1} & = & \text { basis matrix } \\ \Psi_{k} & = & \text { k-th basis function } \\ \Psi_{r} & = & \text { r-th basis function } \\ \Psi_{S}^{T} & = & \text { s-th transpose of the basis function } \\ \omega & = & \text { frequency }\end{array}$

\section{Introduction}

Supporting the Aeronautics Research Mission Directorate guidelines, the National Aeronautics and Space Administration (NASA) Dryden Flight Research Center (DFRC) (Edwards, California) is developing an object-oriented multidisciplinary design, analysis, and optimization (MDAO) tool. ${ }^{1}$ This tool will leverage existing tools and practices, and allow the easy integration and adoption of new state-of-the-art software. At the heart of the object-oriented MDAO tool is the central executive module (CEM) as shown in Fig. 1. In this module, the user will choose an optimization methodology, and provide starting and side constraints for continuous as well as discrete design variables and external file names for interface variables, which communicate between the CEM and each analysis module. The structural analyses modules such as computations of the structural weight, stress, deflection, buckling, and flutter and divergence speeds have been developed and incorporated into the object-oriented MDAO framework.

In general, obtaining aerodynamic influence coefficients (AIC) by direct calculation for integration into preliminary design activities involving disciplines such as aeroelasticity, aeroservoelasticity, and optimization is, at present, a costly and impractical venture. ${ }^{1}$ With the increasing complexity of the configuration and the increasing fidelity of the aerodynamic equations, the computational costs increase rapidly. The unsteady aeroelastic analysis and design optimizations are a challenging task. The time required for unsteady computations whether in frequency domain or time domain will considerably slow down the whole design process. Also, these analyses are usually performed repeatedly to optimize the final design. Even though the computational costs may be reduced by the use of advanced algorithms and improved computer hardware processing speeds, these full aeroelasticity analyses cannot be incorporated effectively within a preliminary design and optimization environment. For example, using the ZAERO ${ }^{\text {TM }}$ (ZONA Technology Incorporated, Scottsdale, Arizona) code to generate the modal AIC matrix for the IKHANA (a modified Predator ${ }^{\circledR}$ B, (General Atomics Aeronautical Systems, Incorporated, San Diego, California) aircraft with existing AIC data, one Mach number, and 16 reduced frequencies, takes an average of 30 minutes on an Intel ${ }^{\circledR}$ Core $^{\mathrm{TM}} 22.80 \mathrm{GHz}$ CPU computer (Intel Corporation, Santa Clara, California). ${ }^{1}$ One of the examples presented in this paper, the HWB aircraft, takes about 50 minutes to generate the modal AICs with existing AIC data.

As a result, there is considerable motivation to be able to perform aeroelastic calculations more quickly and inexpensively using basis function approximation (BFA) method. One of the primary goals behind the current development is to reduce the computation time for generating modal AIC matrices during the optimization procedure.

\section{Basis Function Approximation}

The BFA method requires the unsteady aerodynamics to be represented in the frequency domain. In this study, the BFA for the subsonic and supersonic speeds are discussed. The flutter solution can be found by the classic methods, such as rational function approximation, $\mathrm{k}, \mathrm{p}-\mathrm{k}, \mathrm{p}$, root locus et cetera. A process that 
efficiently incorporates an approximated modal AIC matrix into the MDAO tool for design optimization at a reasonable computational cost has been developed and is outlined in the flowchart shown in Fig. 2.

In linear algebra, a basis is a set of vectors that in a linear combination can represent every vector in a given vector space or free module, and no element of the set can be represented as a linear combination of the others. ${ }^{2}$ In other words, a basis is a linearly independent spanning set.

This paper discusses an effective approach for approximating a modal AIC matrix for optimization with flutter speed constraints. Consider the steps of the approximation process depicted in the flowchart given in Fig. 2. Steps 1 and 2 are performed only once before starting the optimization process. Steps 3, 4, and 5 are done iteratively for aeroelasticity calculation.

In step 1, a set of representative basis functions $\Psi$ is defined and intended to capture salient features of the modal responses the airplane is expected to encounter in the design space. Structural mode shapes obtained from different mass or various stiffness configurations of the airplane can be used as the basis functions. These basis functions are comparison functions ${ }^{3}$ since all the geometric and natural boundary conditions of the airplane are satisfied. Each mode shape of the airplane with the target configuration can be approximated as a linear combination of a set of the basis functions. The target configuration in this paper is simply an arbitrary design point within the design space and is used as a check case.

In step 2 , the representative basis AIC matrices $\tilde{Q}$ are computed corresponding to the representative basis functions defined in step 1 at any Mach number and reduced frequency. These basis AIC matrices are used as input for approximate modal AIC matrix calculation in step 4.

In step 3, for a set of given design structural mode shapes $\Phi$, each mode shape is decomposed in a linear combination of the basis functions. The $i$-th target mode shape vector $\phi(i=1,2, \ldots, m$, where $\mathrm{m}$ is the number of mode shapes) is approximated through the use of a least squares method of the representative basis functions as shown in Eq. (1),

$$
\phi_{i} \approx \sum_{k=1}^{n} \beta_{k}^{i} \psi_{k}
$$

where a vector $\psi_{k}$ is the $k$-th basis function and a scalar coefficient $\beta_{k}^{i}$ is the modal participation factor of the $k$-th basis function on the $i$-th mode shape; $n$ is the number of basis functions.

In step 4, an approximate modal AIC matrix $\bar{Q}$ is computed based on a basis AIC matrix $\tilde{Q}$ and modal participation factors. Let matrices $Q, A$, and $\Phi$ be a modal AIC matrix, a general AIC matrix and a modal matrix, respectively. Then the modal AIC matrix $Q$ can be defined as shown in Eq. (2),

$$
\begin{gathered}
Q=\Phi^{T} A \Phi \\
\text { where } \Phi=\left[\begin{array}{lll}
\phi_{1} & \phi_{2} \cdots \phi_{m}
\end{array}\right]
\end{gathered}
$$

Threfore the $i$-th row and $j$-th column element of the modal AIC matrix $Q$ can be written as shown in Eq. (3),

$$
Q_{i j}=\phi_{i}^{T} A \phi_{j}
$$

Substituting Eq. (1) into Eq. (3) is given in Eq. (4),

$$
\begin{aligned}
Q_{i j}=\phi_{i}^{T} A \phi_{j} & \approx\left(\sum_{k=1}^{n} \beta_{k}^{i} \psi_{k}^{T}\right) A\left(\sum_{k=1}^{n} \beta_{k}^{j} \psi_{k}\right)=\bar{Q}_{i j} \\
\bar{Q}_{i j} & =\sum_{r=1}^{n} \sum_{s=1}^{n} \beta_{s}^{i} \beta_{r}^{j} \psi_{s}^{T} A \psi_{r}
\end{aligned}
$$


The detailed derivations of Eq. (4) are listed in Appendix A. Let the basis AIC matrix $\tilde{Q}$ be defined as shown in Eq. (5),

$$
\tilde{Q}=\Psi^{T} A \Psi
$$

then the $s$-th row and $r$-th column element of the $\tilde{Q}$ can be written as shown in Eq. (6),

$$
\tilde{Q}_{s r}=\psi_{s}^{T} A \psi_{r}
$$

Sustituting Eq. (6) into Eq. (4) gives Eq. (7) for the approximate modal AIC matrix.

$$
\bar{Q}_{i j}=\sum_{r=1}^{n} \sum_{s=1}^{n} \beta_{s}^{i} \beta_{r}^{j} \tilde{Q}_{s r}
$$

The basis AIC matrix $\tilde{Q}$ in Eq. (5) can be computed and saved before starting optimization as mentioned in step 2. During optimization, the mode shapes, $\phi_{i}(i=1,2, \ldots, \mathrm{m})$, are fitted using basis functions $\psi_{k}$ and modal participation factors $\beta_{k}^{i}$ as shown in Eq. (1), and the approximate modal AIC matrix $\bar{Q}$ can be computed using Eq. (7). This approximate modal AIC matrix will be used for flutter analysis in step 5. The resulting flutter speed and frequency could be used for optimization. In step 5, the flutter solution can be found by the classic methods, such as rational function approximation, $\mathrm{k}, \mathrm{p}-\mathrm{k}, \mathrm{p}$, root locus et cetera.

\section{Applications}

In order to validate the proposed BFA technique in subsonic flight regimes, the flutter results using this approximate method are compared with the direct flutter results. First a simple model from the ATW2 program was chosen. Subsequently, a complex practical problem HWB model was analyzed. In this study, structural mode shapes obtained from different mass configurations of the airplane are used as basis functions.

\section{A. A modified Aerostructures Test Wing 2}

The proposed technique has been applied and validated using a modified ATW2. The original ATW2 ${ }^{4}$ test article was actually designed, built, and flight-tested at NASA DFRC as shown in Fig. 3. This wing was cantilevered from a center station pylon and flown on the McDonnell Douglas (now The Boeing Company, Chicago, Illinois) NF-15B test bed aircraft.

In this study, calculated structural mode shapes obtained from different mass configurations of the wing are used as basis functions. To create each different mass configuration, the mass at each mass point is represented using an additional concentrated mass element attached through rigid body elements to the finite element model as shown in Fig. 4. These alterations unintentionally increased the effective wing stiffness and the first natural frequency over that of the actual ATW2 configuration ${ }^{5}$ in spite of the mass increase because of the rigid elements.

The total weight and center of gravity of the various fictitious and target ATW2 mass configurations are listed in Table 1. The direct flutter analysis of the target configuration (mass $=0.0$ units) is performed at Mach 0.82 with 16 reduced frequencies using MSC/NASTRAN (MSC Software Corporation, Santa Ana, California) and ZAERO ${ }^{\mathrm{TM}}$ codes. ${ }^{6,7}$ In order to have better flutter solution convergence for flutter speed, the first 10 structural modes are included in the calculation. While preparing for the approximate flutter solution, three different mass configurations, $0.3,0.6$, and 0.9 units were selected for generating the basis functions.

In order to be able to capture more of the target mode shapes, larger basis functions were generated. Fifteen structural mode shapes for each mass configuration were generated using MSC/NASTRAN. The natural frequencies of the various fictitious and target ATW2 mass configurations are listed in Table 2. 
According to Pak and Lung ${ }^{5}$, the primary natural modes for the first flutter mode are mode numbers 1, 2, and 3; and all of the higher modes are the secondary modes. The first three natural frequencies and mode shapes are the first bending, the first torsion, and the second bending modes, respectively and are shown in Fig. 5. A total of 45 basis functions were generated. Then the 45 by 45 basis AIC matrix corresponding to those 45 basis functions was computed for each of 16 reduced frequencies at Mach 0.82 using ZAERO ${ }^{\text {TM}}$. The mode shapes of the target configuration are fitted using the basis functions together with a least squares method; and then the approximate modal AIC matrices were computed, and the flutter analysis was performed.

For the present test case, ATW2 mode shapes, generated from direct method and BFA method, are plotted in Figs. 5a, 5b and 5c. The approximated mode shapes are very well matched to the target mode shapes. The difference of the first three mode shapes between these two methods is plotted in Fig. 6.

The results of the matched flutter analysis using the direct method and the BFA method are summarized in Table 3. Three percent damping was used for the flutter speed and frequency computation. Both methods predicted the same flutter speed of $543.59 \mathrm{KEAS}$ at a flutter frequency of $49.01 \mathrm{~Hz}$. The speed versus damping, V-g, and speed versus frequency, V- $\omega$ curves from the direct and BFA methods at Mach 0.82 are given in Figs. 7 and 8.

Table 4 lists the computational cost of computing the modal AIC with a given structural mode shape at one Mach number using the direct method and the BFA method. The comparison was done on an Intel ${ }^{\mathbb{B}}$ Core ${ }^{\mathrm{TM}} 2.80 \mathrm{GHz}$ CPU computer. The computational cost of generating the basis functions before the parametric study is not included in the comparison.

\section{B. Hybrid Wing Body Vehicle}

The modified ATW2 is a simple cantilevered wing model and a straight forward application. A hybrid wing body (HWB) aircraft ${ }^{8}$ was selected for a second and more challenging demonstration of the BFA method.

In this HWB application, the basis functions are created based on three different total fuel conditions of the vehicle, configuration number 1,2 , and 3 . The target model weight is bounded by the range of the weight of configurations 2 and 3 . In the finite element model, the various fuel weights are modeled using a concentrated mass element attached directly to each of the node points in the fuel tank area as shown in Fig. 9. ${ }^{9}$ The total weight and center of gravity location of the different fuel weight and target configurations are listed in Table 5.

With target configuration, the direct flutter analysis is performed at Mach 0.50, with 40 structural modes and 14 reduced frequencies using MSC/NASTRAN and ZAERO ${ }^{\mathrm{TM}}$ codes. A classical wing bending torsion type of flutter was predicted in this HWB example. The first fourteen flexible natural frequencies and the first four flexible mode shapes are listed in Table 6 and Fig. 10 respectively. The first four modes are the first symmetric and anti-symmetric bending, and the first symmetric and anti-symmetric torsion.

For the approximate flutter solution, a total of 150 basis functions are generated from 3 different fuel configurations and 50 structural mode shapes for each fuel configuration using MSC/NASTRAN. Then the 150 by 150 basis AIC matrix corresponding to those 150 basis functions is computed for each of 14 reduced frequencies at Mach 0.50 using $\mathrm{ZAERO}^{\mathrm{TM}}$.

The flutter boundary of the HWB at Mach 0.50 using BFA method is compared to that from the direct method. The flutter boundaries are summarized in Table 7. The flutter speed is normalized with respect to direct method flutter speed. With 3-percent structural damping, the normalized flutter speed and the flutter frequency using BFA method are $0.991 \mathrm{~Hz}$ and $2.671 \mathrm{~Hz}$ respectively. The percentage errors of the flutter speed and frequency are 0.86 percent and 0.15 percent. The speed versus damping, V-g, and the speed versus frequency, $\mathrm{V}-\omega$, curves of the HWB at Mach 0.50 obtained from the direct and BFA methods are given in Figs. 11 and 12. The BFA offers consistent flutter speed and frequency predictions on the HWB target configuration. See Table 4 for the computational cost of the HWB example. The computational cost of generating the basis functions and AIC is about 250 minutes in this HWB example. 


\section{Conclusion}

A technique for approximating the modal AIC matrix by using basis functions has been developed and validated, and a process for using the resulting AIC matrix in aeroelastic analysis and design optimization has been proposed. The approximation method has been applied to the aeroelastic analyses, and the results are essentially identical to those using direct solution. The technique presented has been shown to offer consistent flutter speed prediction on an ATW2 configuration and a HWB type vehicle with a negligibly small loss in precision. These results may have practical significance in the analysis of aircraft aeroelastic calculation and could lead to a more efficient design optimization cycle. The basis function approximation approach yields significant improvements in computational efficiency as compared to the original approach, thereby meeting the objective of this study.

\section{Appendix A}

$$
\begin{aligned}
& \bar{Q}_{i j}=\left(\sum_{k=1}^{n} \beta_{k}^{i} \psi_{k}^{T}\right) A\left(\sum_{k=1}^{n} \beta_{k}^{j} \psi_{k}\right) \\
& =\left(\sum_{k=1}^{n} \beta_{k}^{i} \psi_{k}^{T}\right) A\left(\beta_{1}^{j} \psi_{1}+\beta_{2}^{j} \psi_{2}+\cdots+\beta_{n}^{j} \psi_{n}\right) \\
& =\left(\sum_{k=1}^{n} \beta_{k}^{i} \psi_{k}^{T}\right)\left(A \beta_{1}^{j} \psi_{1}+A \beta_{2}^{j} \psi_{2}+\cdots+A \beta_{n}^{j} \psi_{n}\right) \\
& =\left(\sum_{k=1}^{n} \beta_{k}^{i} \psi_{k}^{T}\right)\left(\beta_{1}^{j} A \psi_{1}+\beta_{2}^{j} A \psi_{2}+\cdots+\beta_{n}^{j} A \psi_{n}\right) \\
& =\left(\beta_{1}^{i} \psi_{1}^{T}+\beta_{2}^{i} \psi_{2}^{T}+\cdots+\beta_{n}^{i} \psi_{n}^{T}\right)\left(\beta_{1}^{j} A \psi_{1}+\beta_{2}^{j} A \psi_{2}+\cdots+\beta_{n}^{j} A \psi_{n}\right) \\
& =\beta_{1}^{i} \psi_{1}^{T} \beta_{1}^{j} A \psi_{1}+\beta_{2}^{i} \psi_{2}^{T} \beta_{1}^{j} A \psi_{1}+\cdots+\beta_{n}^{i} \psi_{n}^{T} \beta_{1}^{j} A \psi_{1} \\
& +\beta_{1}^{i} \psi_{1}^{T} \beta_{2}^{j} A \psi_{2}+\beta_{2}^{i} \psi_{2}^{T} \beta_{2}^{j} A \psi_{2}+\cdots+\beta_{n}^{i} \psi_{n}^{T} \beta_{2}^{j} A \psi_{2}+\cdots+ \\
& +\beta_{1}^{i} \psi_{1}^{T} \beta_{n}^{j} A \psi_{n}+\beta_{2}^{i} \psi_{2}^{T} \beta_{n}^{j} A \psi_{n}+\cdots+\beta_{n}^{i} \psi_{n}^{T} \beta_{n}^{j} A \psi_{n} \\
& =\beta_{1}^{i} \beta_{1}^{j} \psi_{1}^{T} A \psi_{1}+\beta_{2}^{i} \beta_{1}^{j} \psi_{2}^{T} A \psi_{1}+\cdots+\beta_{n}^{i} \beta_{1}^{j} \psi_{n}^{T} A \psi_{1} \\
& +\beta_{1}^{i} \beta_{2}^{j} \psi_{1}^{T} A \psi_{2}+\beta_{2}^{i} \beta_{2}^{j} \psi_{2}^{T} A \psi_{2}+\cdots+\beta_{n}^{i} \beta_{2}^{j} \psi_{n}^{T} A \psi_{2}+\cdots+ \\
& +\beta_{1}^{i} \beta_{n}^{j} \psi_{1}^{T} A \psi_{n}+\beta_{2}^{i} \beta_{n}^{j} \psi_{2}^{T} A \psi_{n}+\cdots+\beta_{n}^{i} \beta_{n}^{j} \psi_{n}^{T} A \psi_{n} \\
& =\beta_{1}^{i} \beta_{1}^{j} \psi_{1}^{T} A \psi_{1}+\beta_{1}^{i} \beta_{2}^{j} \psi_{1}^{T} A \psi_{2}+\cdots+\beta_{1}^{i} \beta_{n}^{j} \psi_{1}^{T} A \psi_{n} \\
& +\beta_{2}^{i} \beta_{1}^{j} \psi_{2}^{T} A \psi_{1}+\beta_{2}^{i} \beta_{2}^{j} \psi_{2}^{T} A \psi_{2}+\cdots+\beta_{2}^{i} \beta_{n}^{j} \psi_{2}^{T} A \psi_{n}+\cdots+ \\
& +\beta_{n}^{i} \beta_{1}^{j} \psi_{n}^{T} A \psi_{1}+\beta_{n}^{i} \beta_{2}^{j} \psi_{n}^{T} A \psi_{2}+\cdots+\beta_{n}^{i} \beta_{n}^{j} \psi_{n}^{T} A \psi_{n} \\
& \bar{Q}_{i j}=\sum_{r=1}^{n} \sum_{s=1}^{n} \beta_{s}^{i} \beta_{r}^{j} \psi_{s}^{T} A \psi_{r} \\
&
\end{aligned}
$$




\section{References}

${ }^{1}$ Pak, C., and Li, W., "Multidisciplinary Design, Analysis and Optimization Tool Development Using a Genetic Algorithm," Proceedings of the $26^{\text {th }}$ Congress of International Council of the Aeronautical Sciences, Anchorage, Alaska, 2008.

${ }^{2}$ Kahaner, D., Moler, C., and Nash, S., Numerical Methods and Software, Prentice-Hall, Englewood Cliffs, New Jersey, 1989.

${ }^{3}$ Meirovitch, L., Analytical Methods in Vibrations, Macmillan Series in Applied Mechanics, The Macmillan Company, New York, 1967.

${ }^{4}$ Lung, S., and Pak, C.,"Updating the Finite Element Model of the Aerostructures Test Wing Using Ground Vibration Test Data," AIAA-2009-2528, Proceedings of the 50th AIAA/ASME/ASCE/AHS/ASC Structures, Structural Dynamics, and Materials Conference, Palm Springs, California, 2009.

${ }^{5}$ Pak, C., and Lung, S., "Reduced Uncertainties in the Robust Flutter Analysis of the Aerostructures Test Wing," Proceedings of the $27^{\text {th }}$ Congress of International Council of the Aeronautical Sciences, Nice, France, 2010 (to be published).

${ }^{6}$ ZAERO Theoretical Manual, ZONA Technology, Incorporated, 2007.

${ }^{7}$ Reymond, M., and Miller, M., MSC/NASTRAN Quick Reference Guide version 69, The MacNeal-Schwendler Corporation, 1996.

${ }^{8}$ Felder, J., Kim, H. and Brown, G., "Turboelectric Distributed Propulsion Engine Cycle Analysis for Hybrid-WingBody Aircraft," AIAA-2009-1132, Proceedings of the 47th AIAA Aerospace Sciences Meeting, Orlando, Florida, 2009.

${ }^{9}$ Velicki, A., "Damage Arresting Composites for Shaped Vehicles - Phase 1 Final Report," NASA/CR-2009-215932, 2009.

Tables

Table 1. Summary of total weight and center of gravity location for the ATW2 with different fictitious mass configurations.

\begin{tabular}{lcccc}
\hline \hline \multicolumn{1}{c}{ Mass configuration } & 1 & 2 & 3 & Target \\
\hline Total weight, lb & 4.046 & 5.250 & 6.450 & 2.850 \\
X center of gravity, in & 14.510 & 14.950 & 15.220 & 13.710 \\
Y center of gravity, in & -8.760 & -9.010 & -9.170 & -8.290 \\
Z center of gravity, in & 0.000 & 0.000 & 0.000 & 0.000 \\
Number of modes & 15 & 15 & 15 & 10 \\
\hline \hline
\end{tabular}


Table 2. Summary of natural frequencies $(\mathrm{Hz})$ of the ATW2 with different fictitious mass configurations.

\begin{tabular}{ccccc}
\hline \hline Mode & Configuration 1 & Configuration 2 & Configuration 3 & Target \\
\hline 1 & 18.10 & 15.98 & 14.45 & 21.32 \\
2 & 62.81 & 61.91 & 60.60 & 63.55 \\
3 & 85.87 & 77.10 & 71.84 & 103.18 \\
4 & 126.60 & 112.29 & 101.86 & 147.79 \\
5 & 191.68 & 173.89 & 160.47 & 208.55 \\
6 & 230.10 & 220.25 & 215.18 & 265.53 \\
7 & 357.83 & 317.80 & 290.89 & 408.75 \\
8 & 403.33 & 383.13 & 367.52 & 434.86 \\
9 & 450.24 & 444.21 & 426.69 & 472.85 \\
10 & 532.04 & 477.94 & 448.37 & 589.94 \\
11 & 628.72 & 616.37 & 600.44 & \\
12 & 633.07 & 630.08 & 628.51 & \\
13 & 708.57 & 707.15 & 684.02 & \\
14 & 729.18 & 726.37 & 705.74 & \\
15 & 782.46 & 728.68 & 725.76 & \\
\hline \hline
\end{tabular}

Table 3. Summary of the ATW2 flutter results comparison for BFA and direct method.

\begin{tabular}{lccc}
\hline \hline & BFA method & Direct method & Error, \% \\
\hline Flutter speed, KEAS & 543.59 & 543.59 & 0.0 \\
Flutter frequency, Hz & 49.01 & 49.01 & 0.0 \\
\hline \hline
\end{tabular}

Table 4. Computational cost comparison of BFA and direct method.

\begin{tabular}{lcc}
\hline \hline \multicolumn{1}{c}{ Test case } & ATW2 & HWB \\
\hline Number of nodes & 269 & $\sim 24000$ \\
Number of structural modes & 10 & 40 \\
Number of reduced & 16 & 14 \\
frequencies & & \\
Direct method elapsed time & $43 \mathrm{~s}$ & $50 \mathrm{~min}$ \\
BFA method elapsed time & $8 \mathrm{~s}$ & $9 \mathrm{~min}$ \\
\hline \hline
\end{tabular}


Table 5. Different fuel weight configurations versus target fuel weight configurations of the HWB.

\begin{tabular}{lcccc}
\hline \hline Mass configuration & 1 & 2 & 3 & Target \\
\hline Total weight, lb & 403781 & 478941 & 591681 & 535311 \\
X center of gravity, in & 1337.60 & 1334.51 & 1331.34 & 1332.76 \\
Y center of gravity, in & 0.75 & 0.46 & 0.16 & 0.29 \\
Z center of gravity, in & 20.89 & 21.35 & 21.82 & 21.61 \\
Number of modes & 50 & 50 & 50 & 40 \\
\hline \hline
\end{tabular}

Table 6. Natural frequencies $(\mathrm{Hz})$ of the HWB with various fuel weight and target fuel weight configurations.

\begin{tabular}{ccccc}
\hline Mode & Configuration 1 & Configuration 2 & Configuration 3 & Target \\
\hline 1 & 1.60 & 1.54 & 1.47 & 1.50 \\
2 & 1.74 & 1.64 & 1.53 & 1.58 \\
3 & 3.52 & 3.35 & 3.13 & 3.24 \\
4 & 3.64 & 3.45 & 3.22 & 3.33 \\
5 & 5.24 & 5.00 & 4.54 & 4.83 \\
6 & 5.26 & 5.04 & 4.54 & 4.89 \\
7 & 6.42 & 5.42 & 4.68 & 4.92 \\
8 & 6.42 & 5.43 & 4.76 & 4.92 \\
9 & 6.92 & 6.66 & 5.81 & 6.31 \\
10 & 7.12 & 6.68 & 5.82 & 6.31 \\
11 & 7.33 & 6.95 & 6.11 & 6.39 \\
12 & 7.65 & 6.95 & 6.32 & 6.48 \\
13 & 8.22 & 7.03 & 6.69 & 6.85 \\
14 & 8.23 & 7.40 & 7.06 & 7.22 \\
\hline \hline
\end{tabular}

Table 7. Summary of the HWB flutter results comparison for BFA and direct method.

\begin{tabular}{lccc}
\hline & BFA method & Direct method & Error, \% \\
\hline Flutter speed, normalized & 0.991 & 1.000 & 0.86 \\
Flutter frequency, Hz & 2.671 & 2.667 & 0.15 \\
\hline \hline
\end{tabular}


Figures

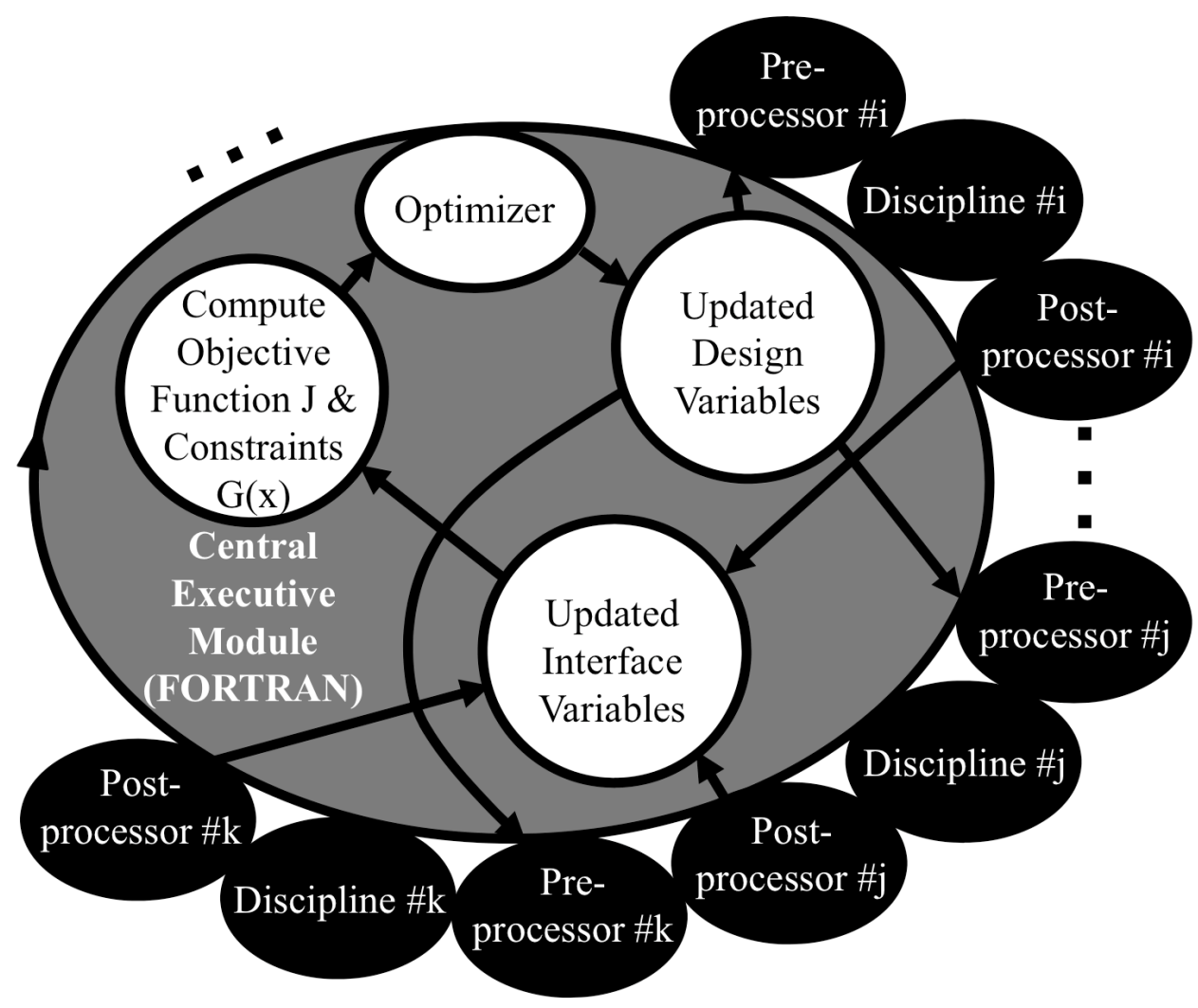

Figure 1. Object-oriented multidisciplinary design, analysis, and optimization tool.

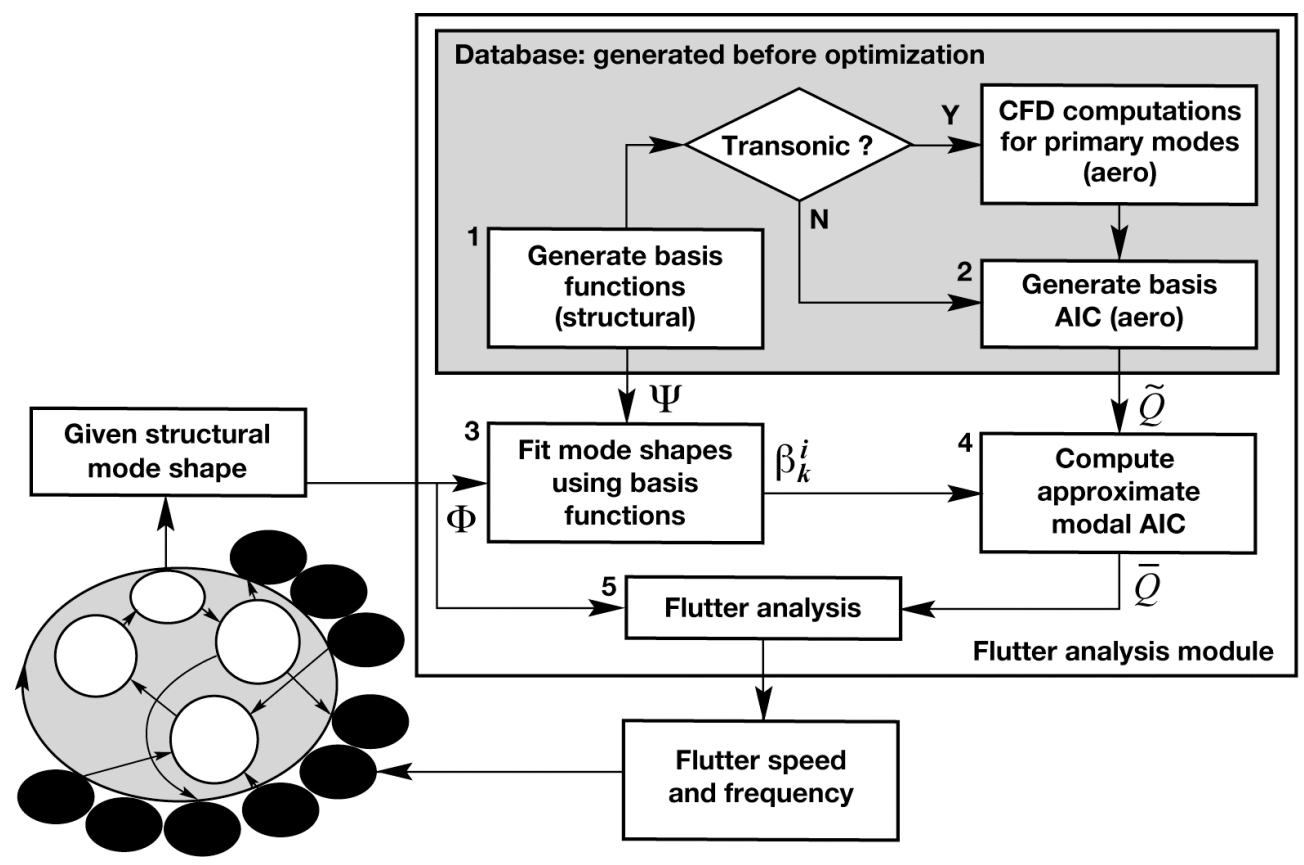

Figure 2. Flowchart of the flutter analysis module in object-oriented MDAO tool. 


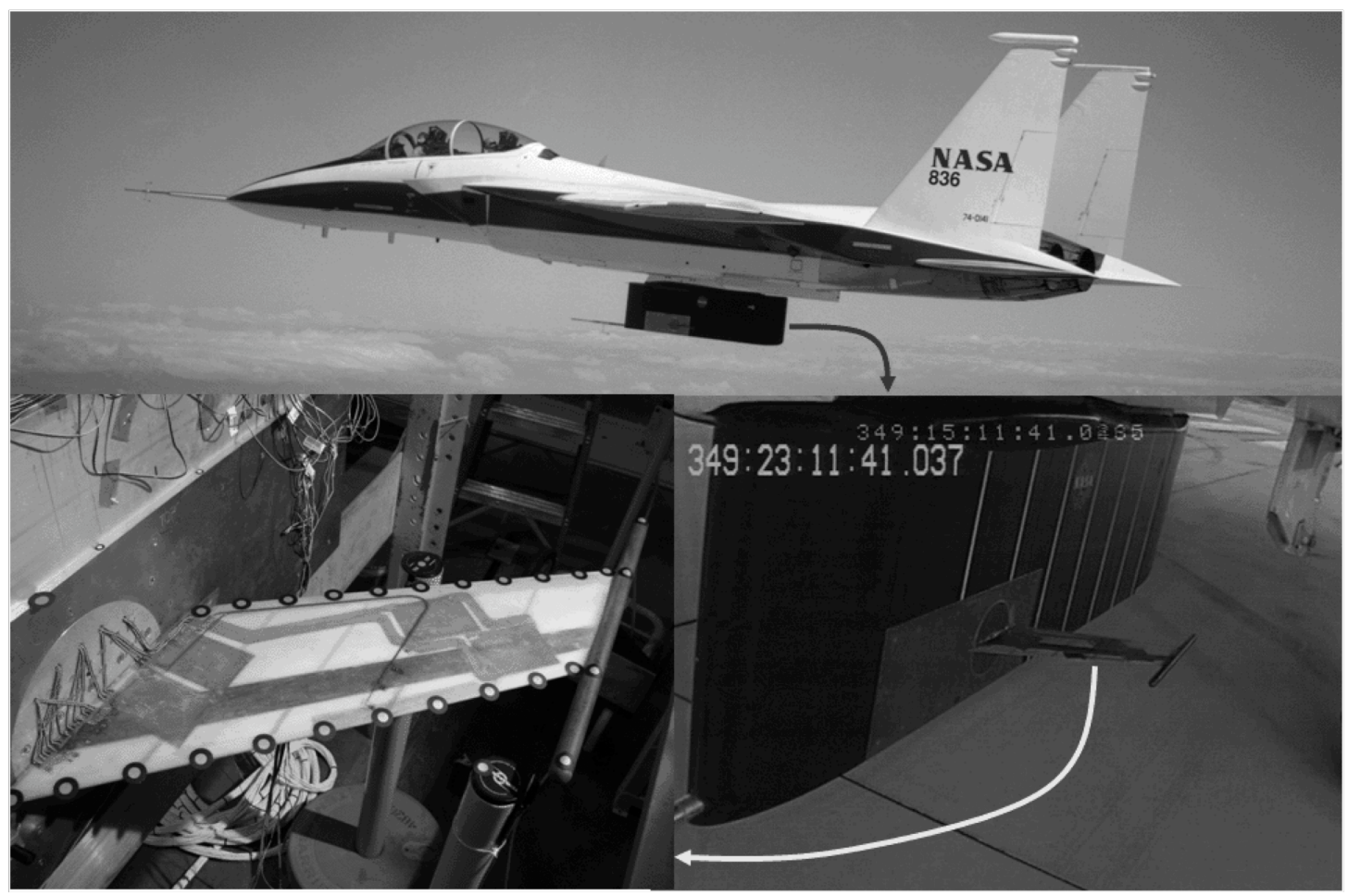

Figure 3. Aerostructures test wing 2.

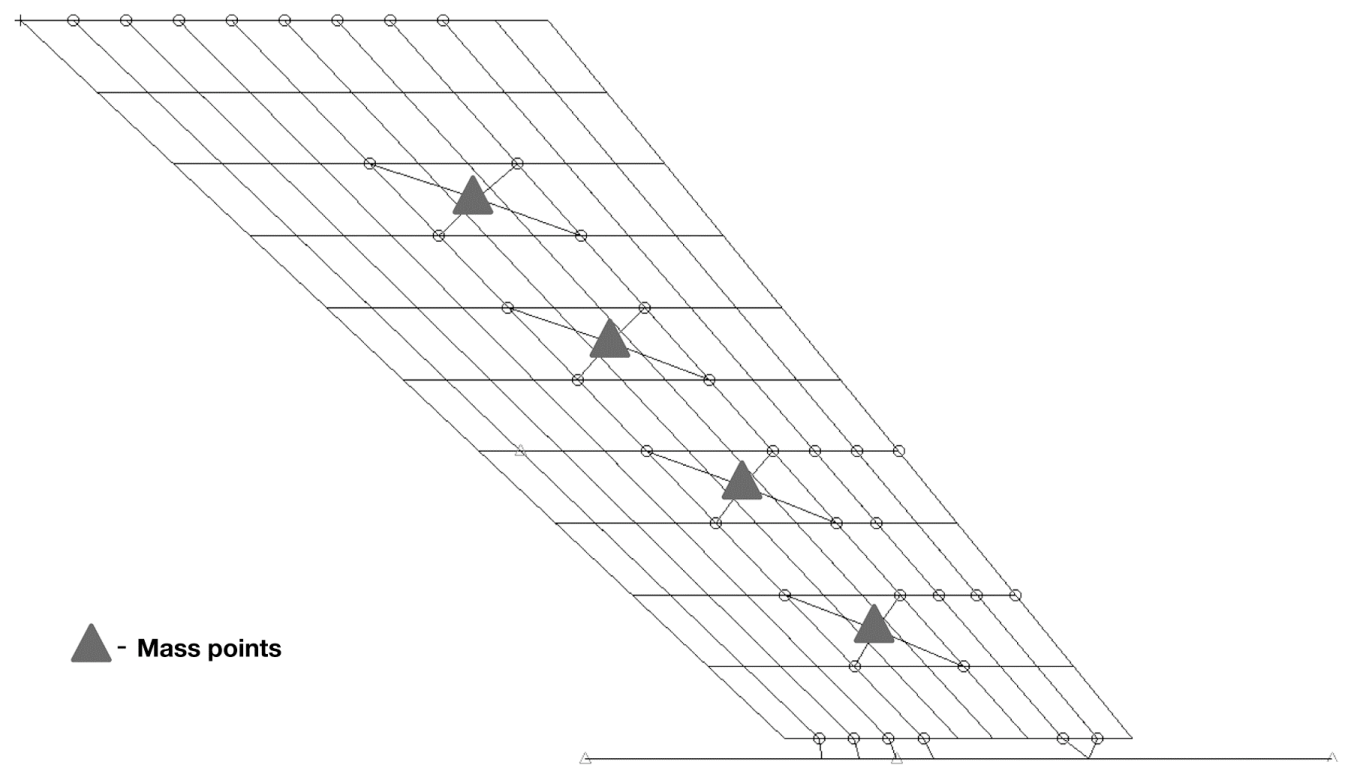

Figure 4. Aerostructures test wing 2 finite element model with fictitious mass point locations. 


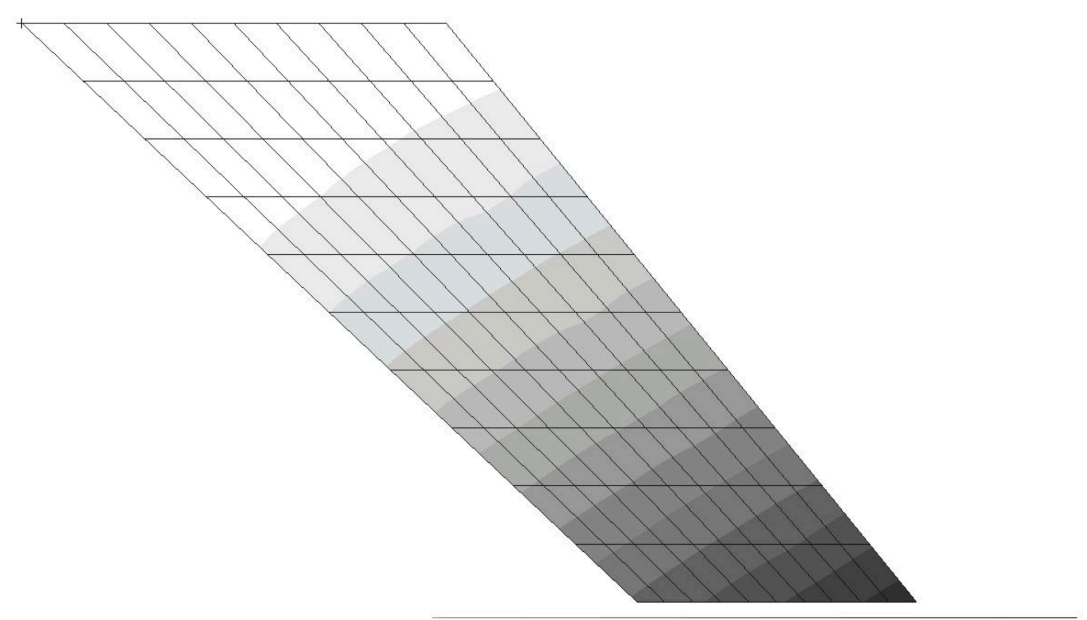

a) Mode 1 (First bending), $21.32 \mathrm{~Hz}$

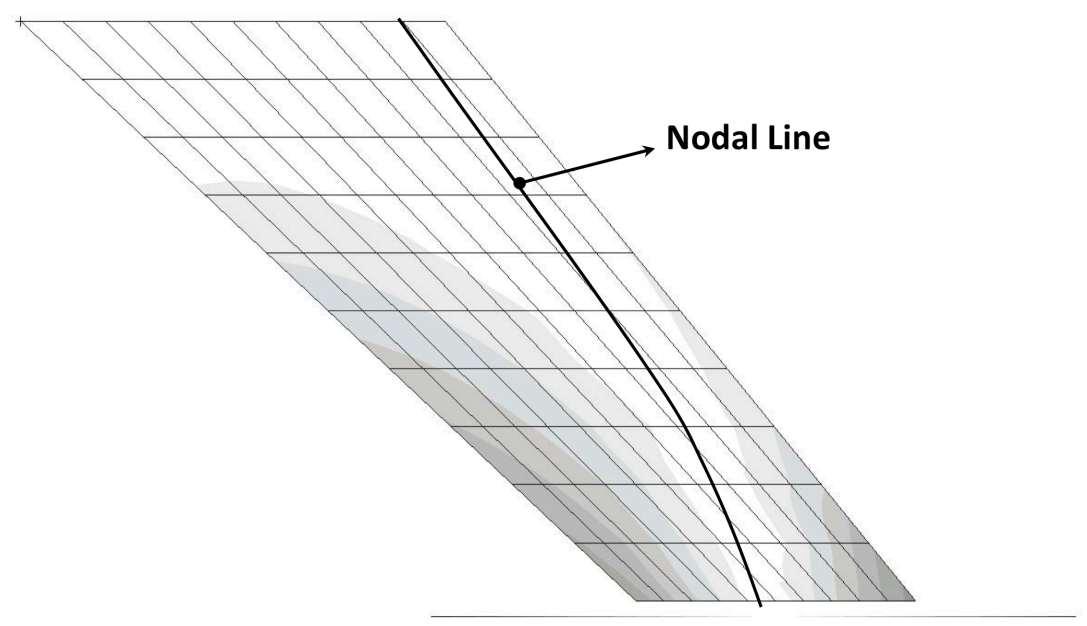

b) Mode 2 (Second bending and first torsion), $63.55 \mathrm{~Hz}$.

Figure 5. Structural finite element model mode shapes and frequencies of the ATW2 with target mass configuration (direct method). 


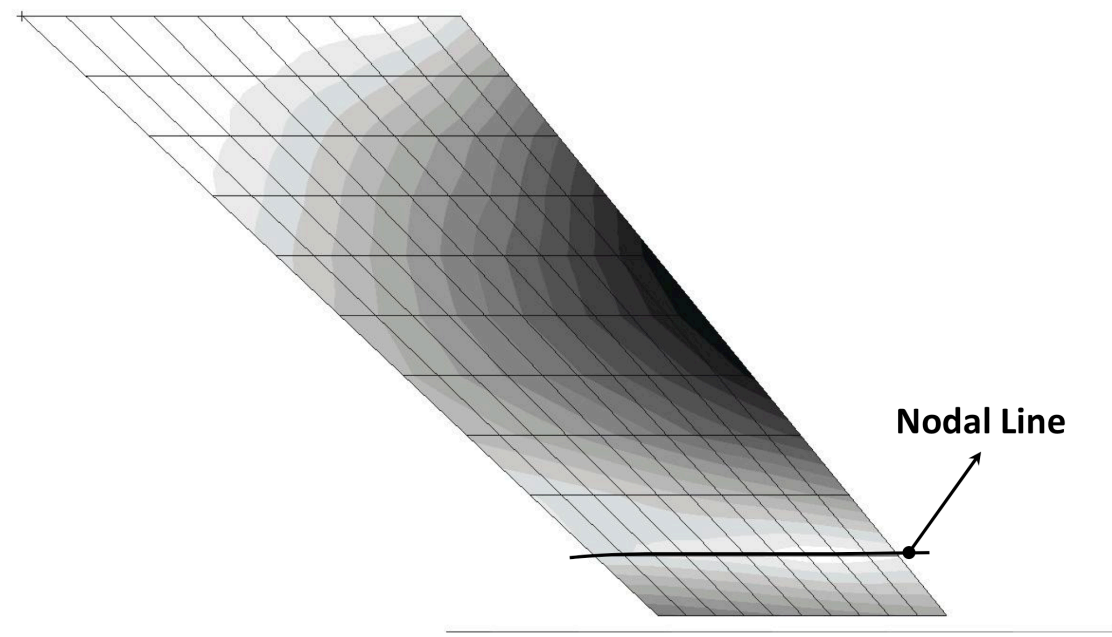

c) Mode 3 (Second bending and first torsion), $103.18 \mathrm{~Hz}$.

Figure 5. Concluded.

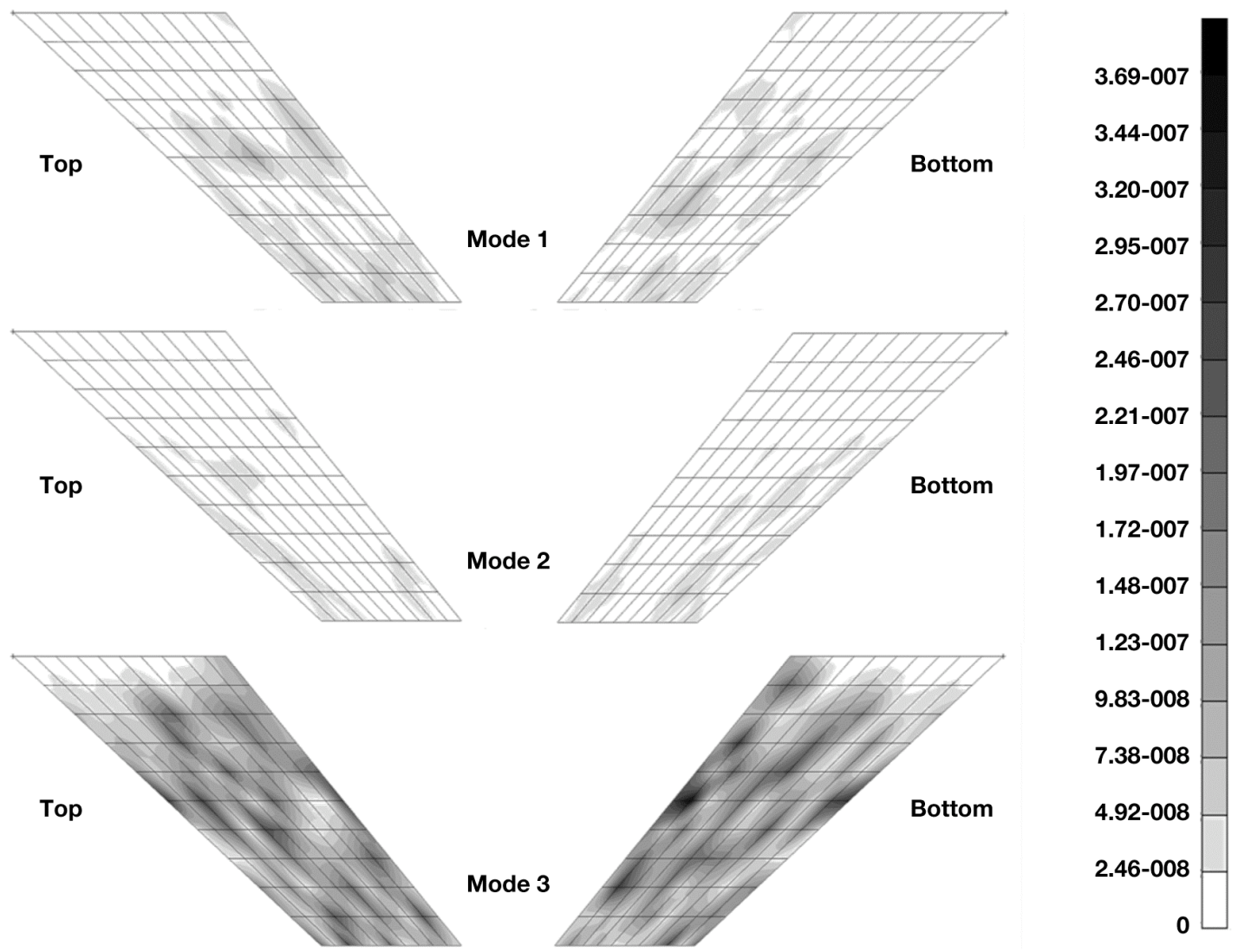

Figure 6. Structural finite element model mode shapes error of the ATW2 with target mass configuration. 


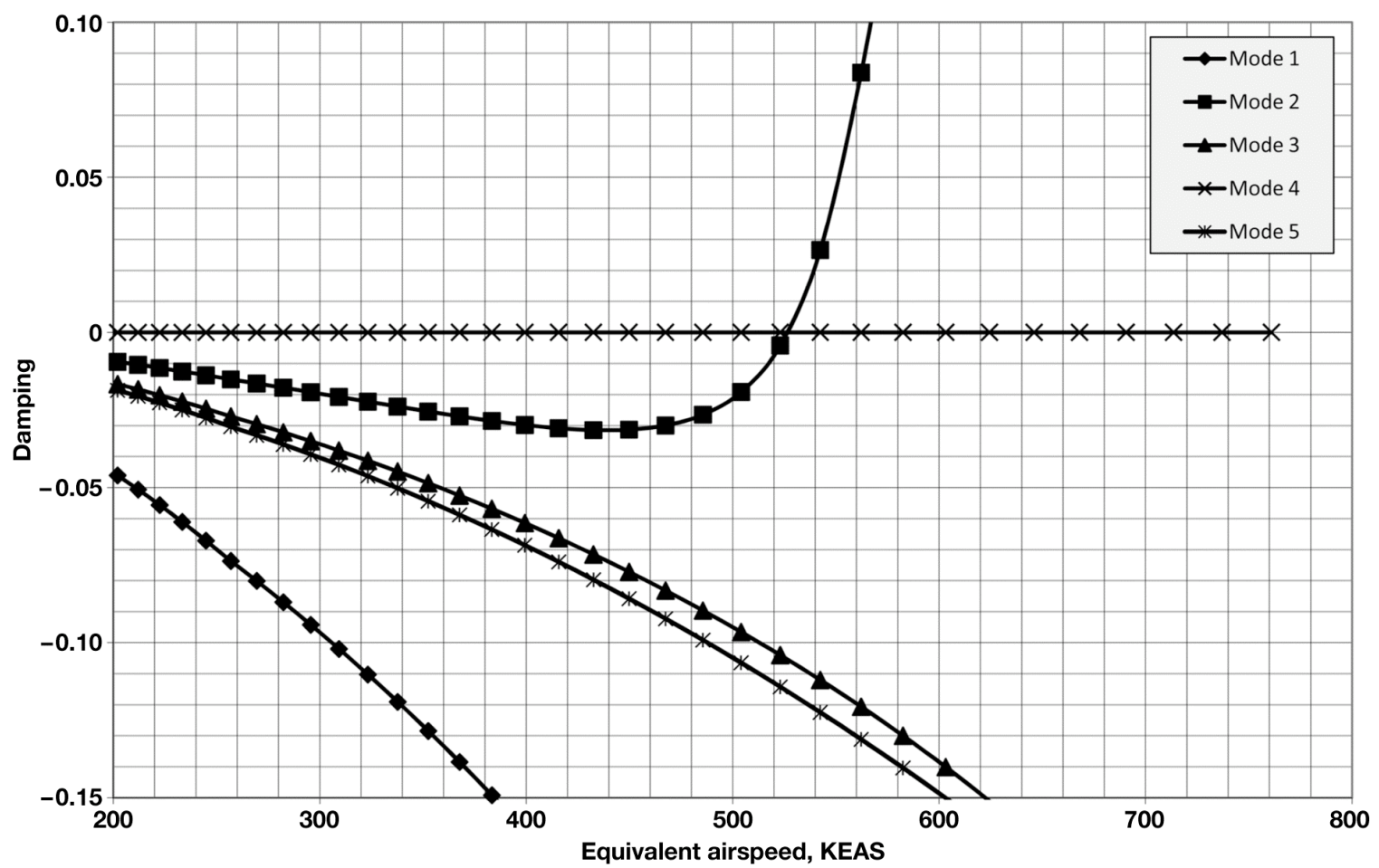

a) V-g plot

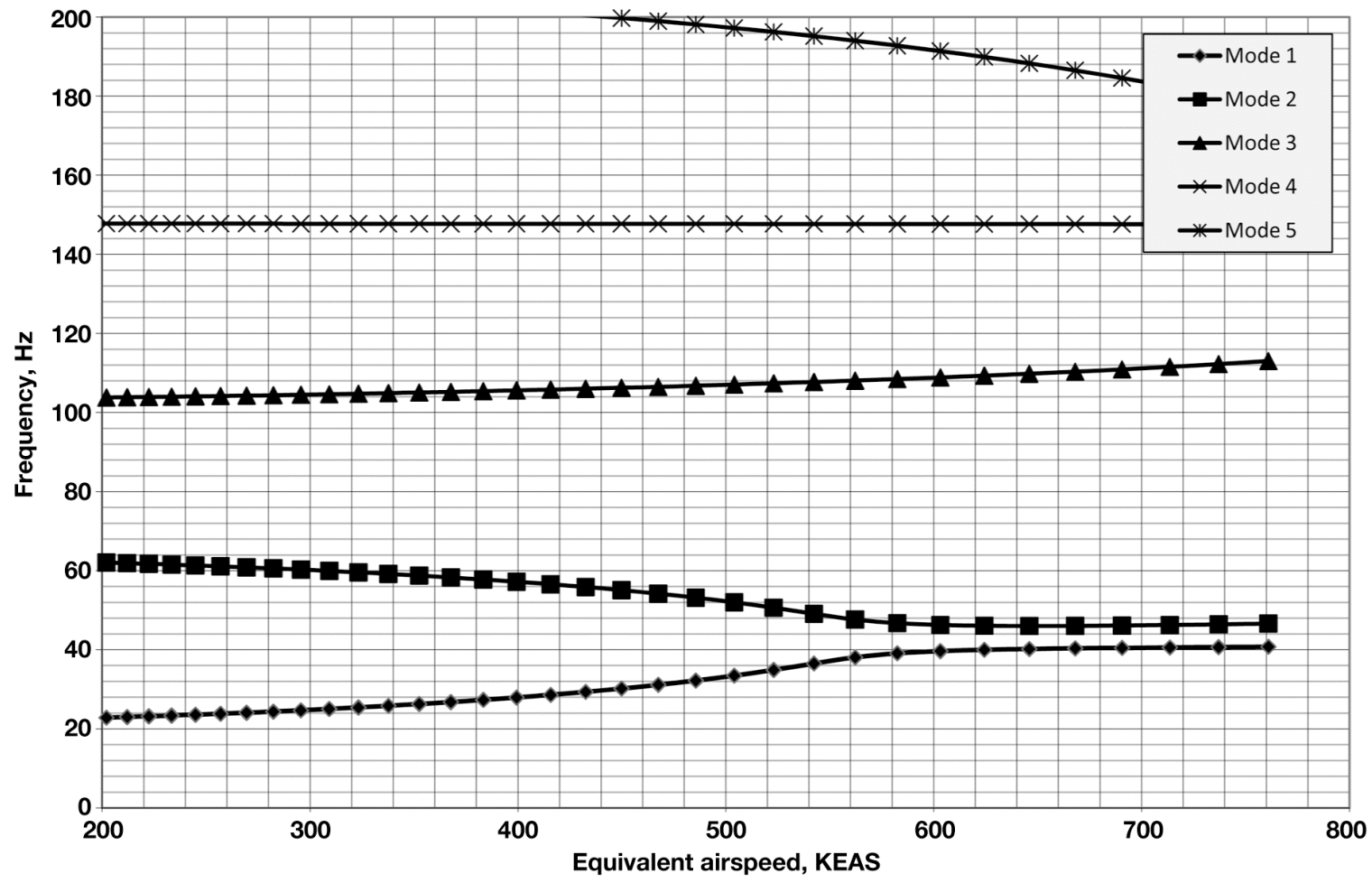

b) $\mathrm{V}-\omega$ plot

Figure 7. V-g and V- $\omega$ plots for the ATW2 at Mach 0.82 using direct modal AIC matrices. 


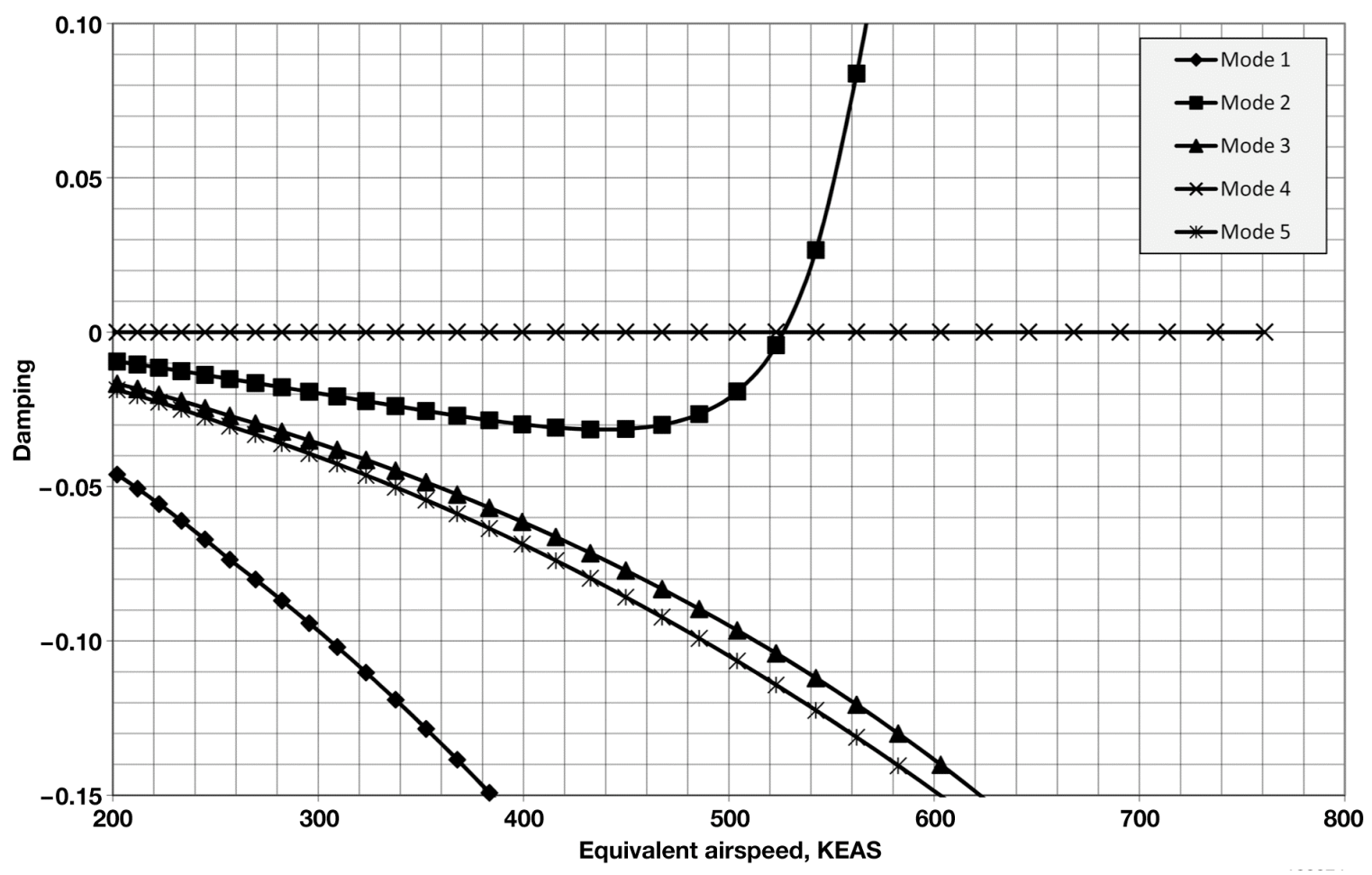

a) V-g plot

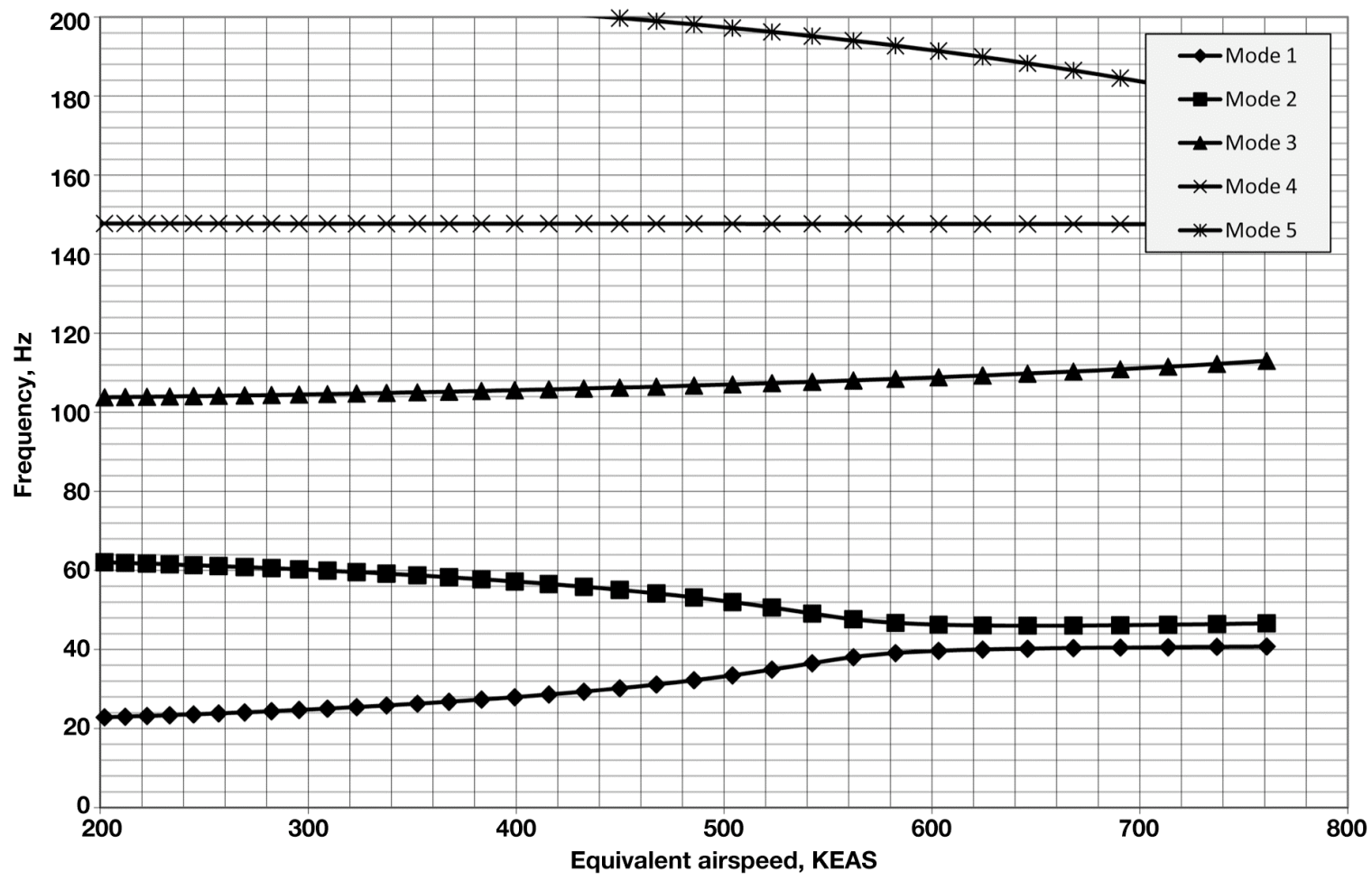

b) $\mathrm{V}-\omega$ plot

Figure 8. V-g and V- $\omega$ plots for the ATW2 at Mach 0.82 using approximate modal AIC matrices. 


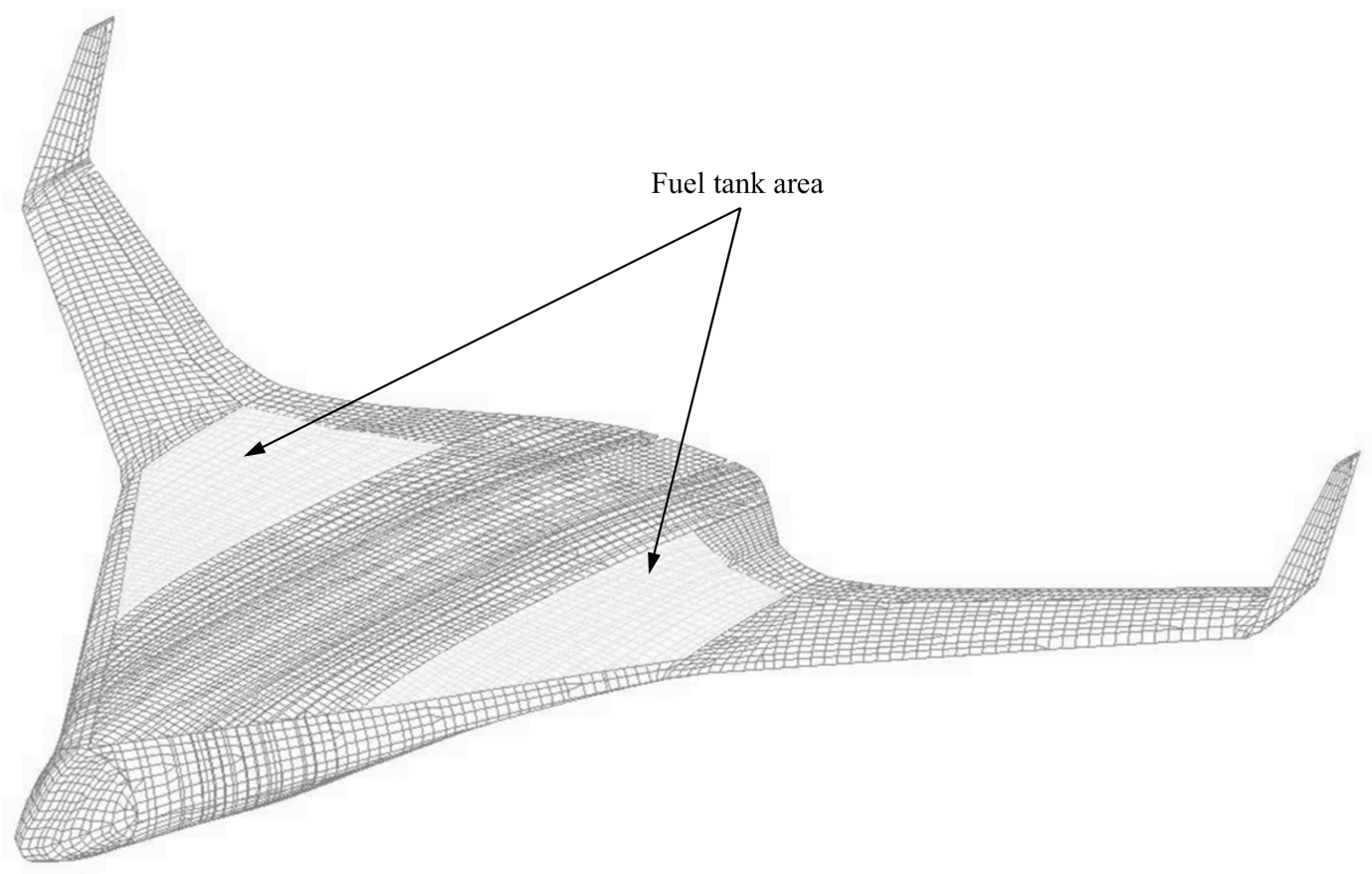

Figure 9. Hybrid wing body aircraft finite element model.

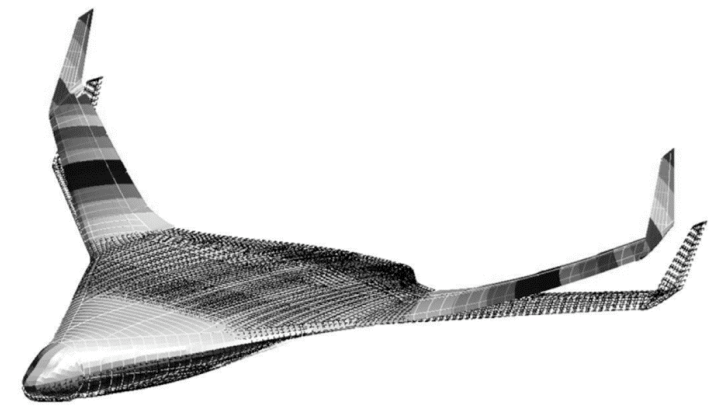

Flexible Mode 1 (First symmetric bending)

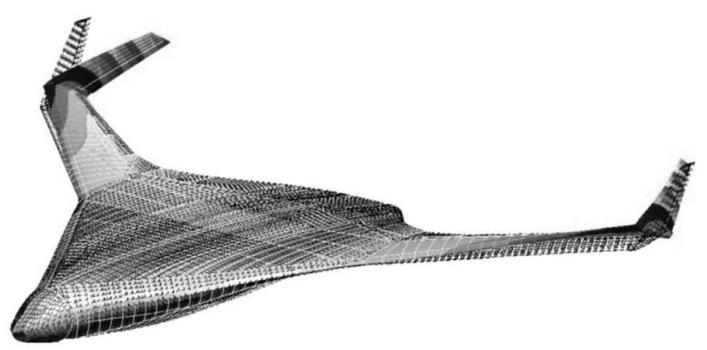

Flexible Mode 3 (First symmetric torsion)

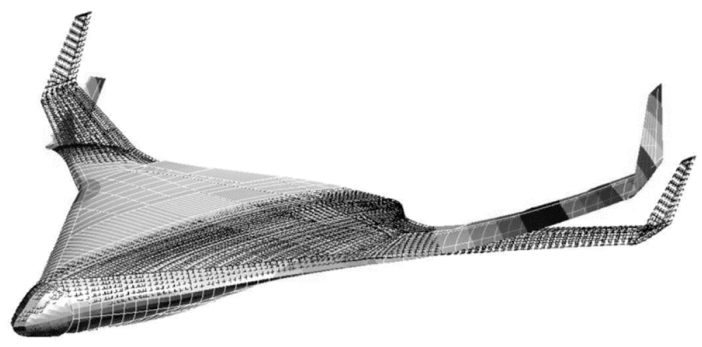

Flexible Mode 2 (First anti-symmetric bending)

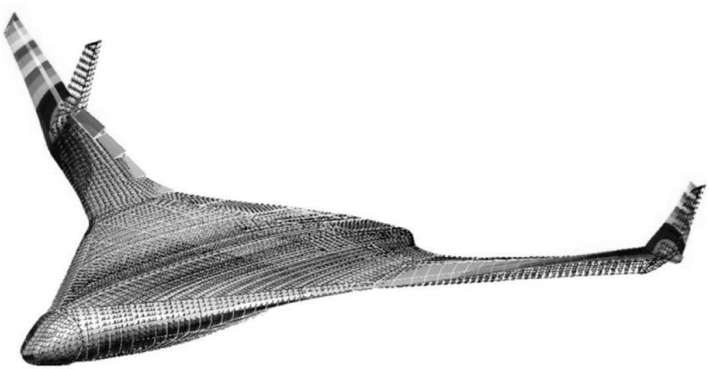

Flexible Mode 4 (First anti-symmetric torsinn)

Figure 10. Hybrid wing body aircraft mode shapes. 


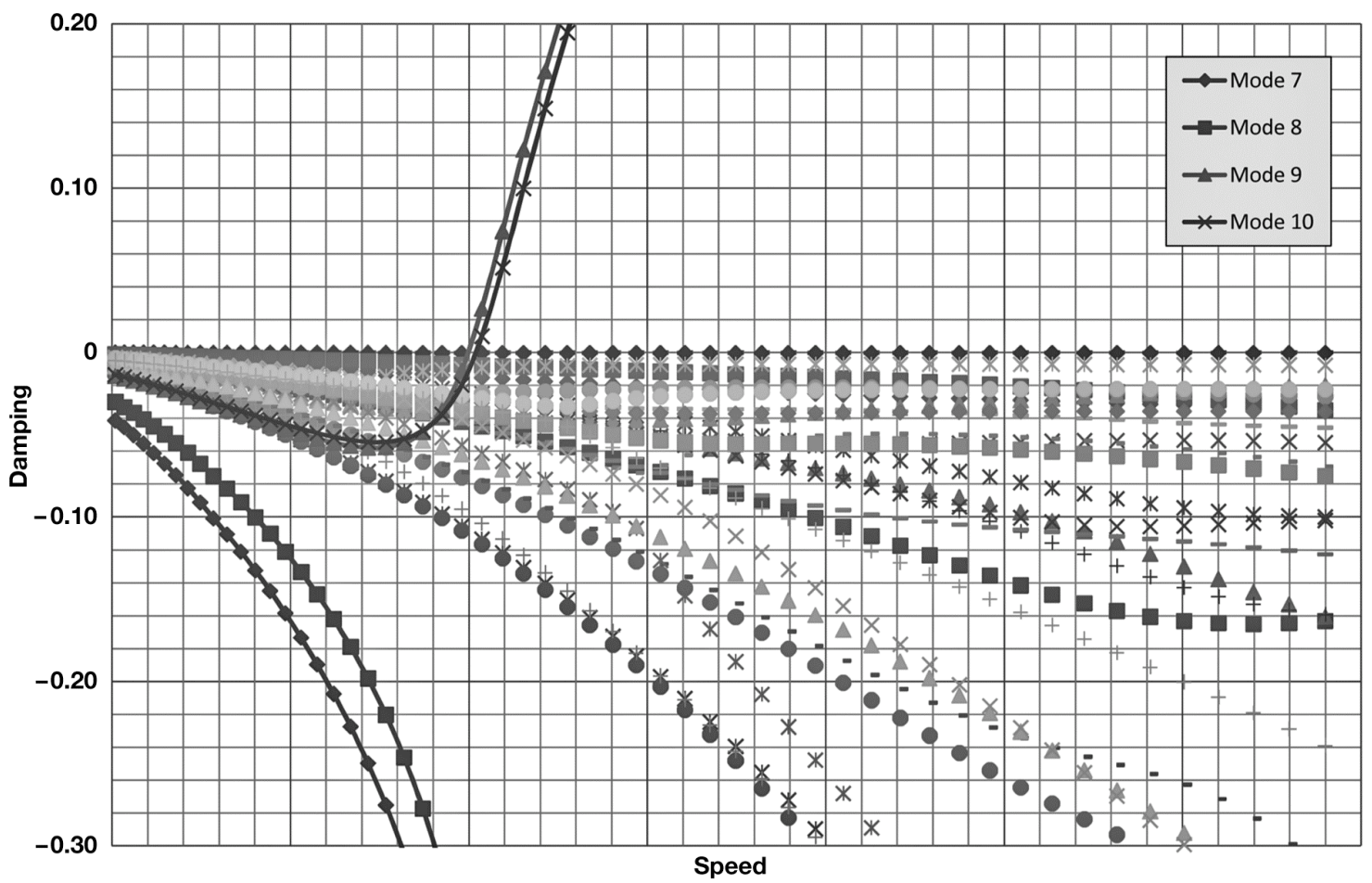

a) V-g plot

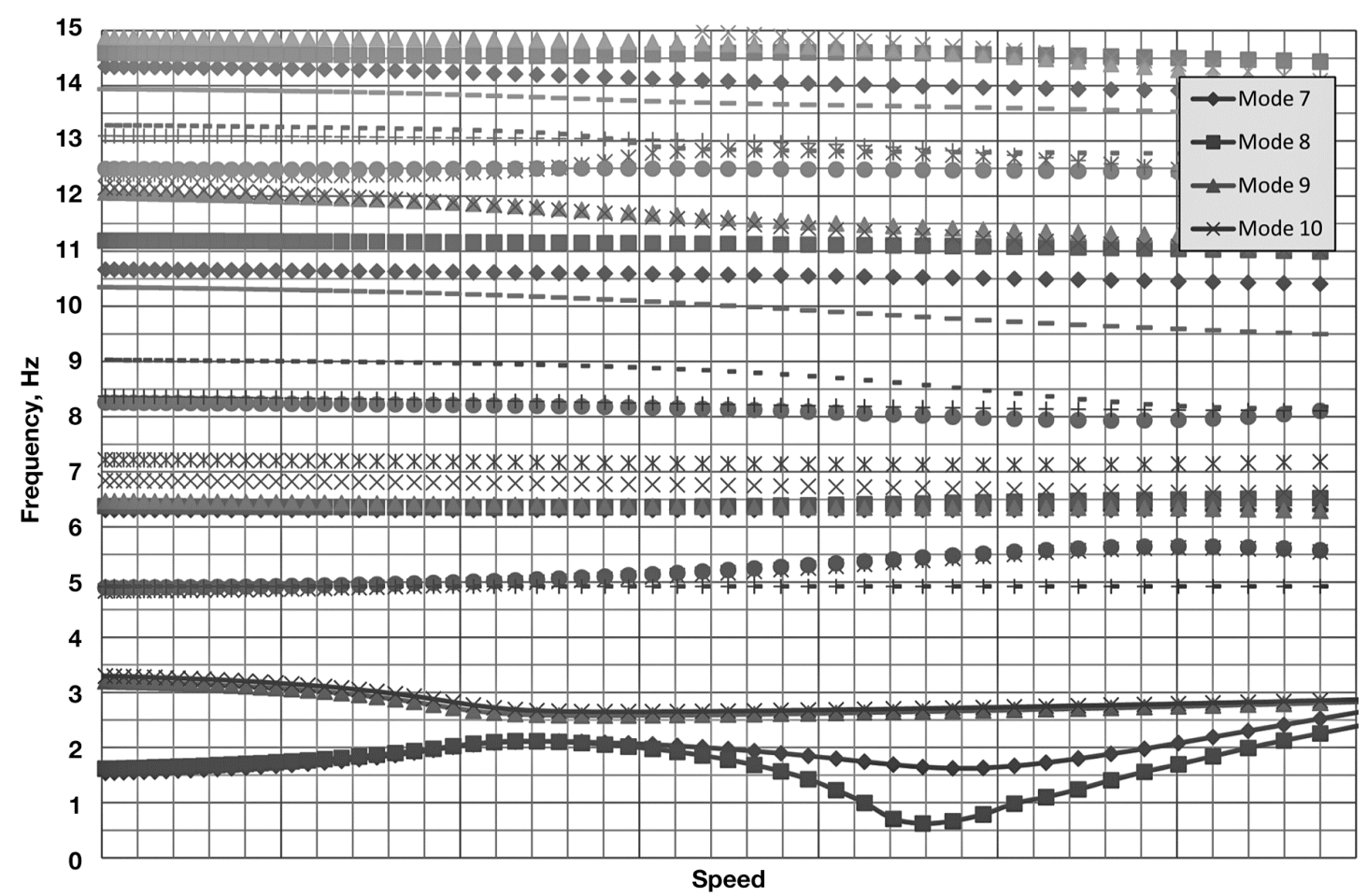

b) $\mathrm{V}-\omega$ plot

Figure 11. V-g and V- $\omega$ plots for the HWB at Mach 0.50 using direct modal AIC matrices. 


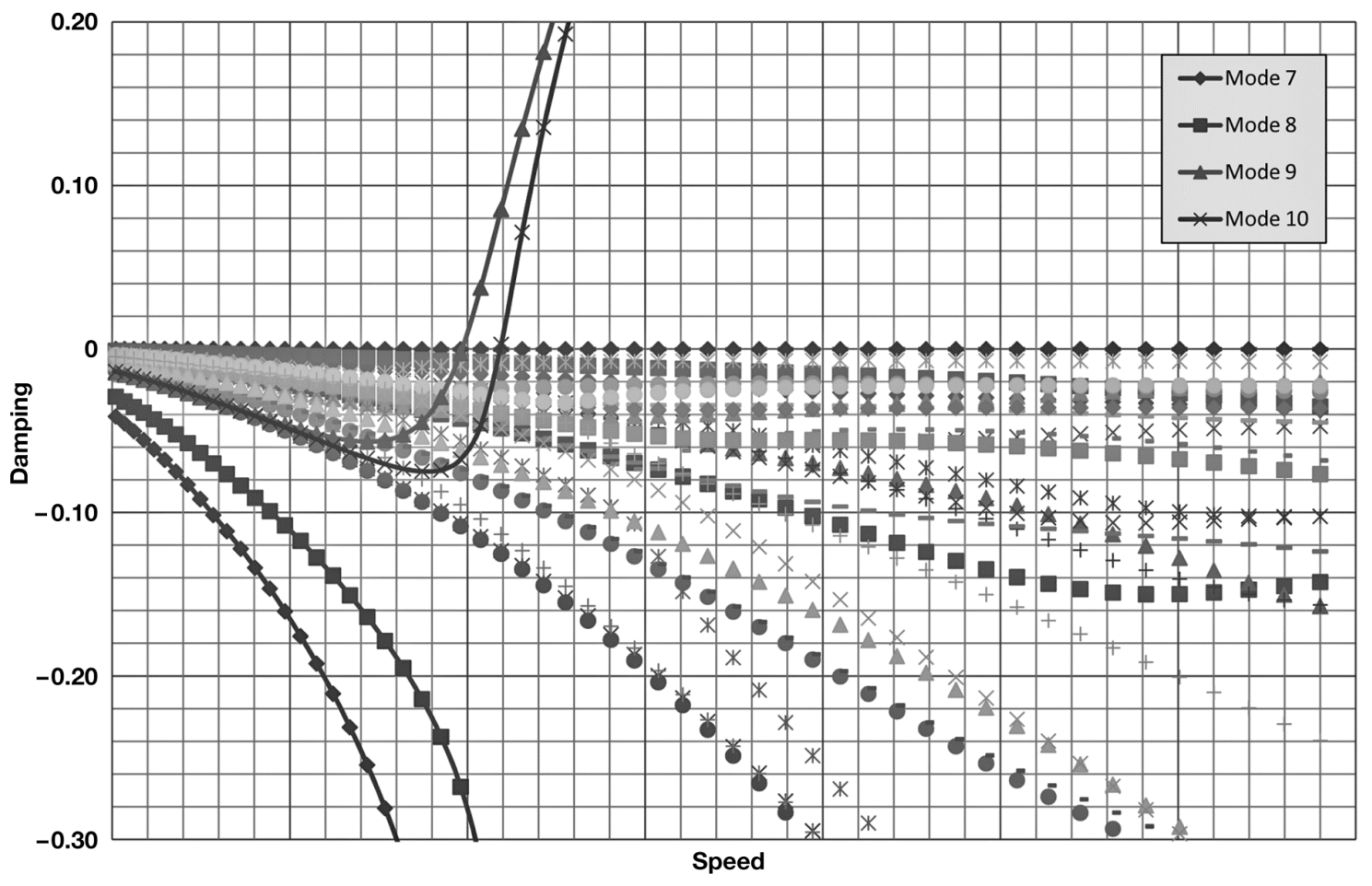

a) V-g plot

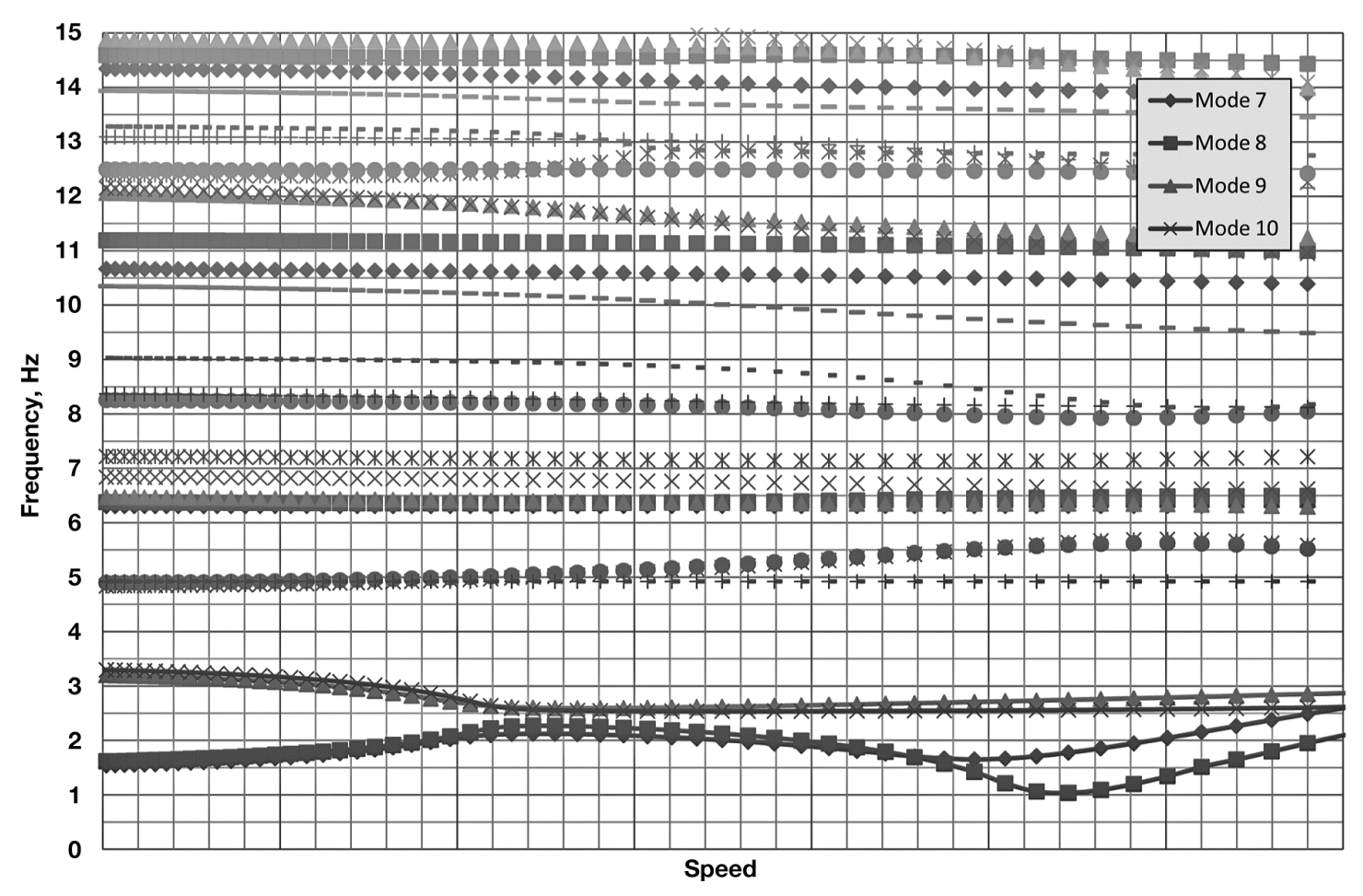

b) V- $\omega$ plot

Figure 12. V-g and V- $\omega$ plots for the HWB at Mach 0.50 using approximate modal AIC matrices. 


\title{
Application of Approximate Unsteady Aerodynamics for Flutter Analysis
}

\author{
Wesley W. Li \& Chan-gi Pak, Ph.D.
}

Structural Dynamics Group, Aerostructures Branch (Code RS)

NASA Dryden Flight Research Center 


\section{Agenda}

Introductions

Objectives

Basis Function Approximation

- Validations \& Applications

- A Modified Aerostructures Test Wing (ATW) 2

- A Hybrid Wing Body (HWB) Aircraft

口 Computational Cost Comparison

$\square$ Conclusions 


\section{Introductions}

Supporting the Aeronautics Research Mission Directorate (ARMD) guidelines, the NASA Dryden has developed an Object-Oriented Optimization $\left(\mathrm{O}^{3}\right)$ tool. This tool leverages existing tools and practices, and allow the easy integration and adoption of new state-of-the-art software.

* Choose an optimization methodology.

* Define objective and constraint functions from performance indices

* Provide starting and side constraints for continuous as well as discrete design variables and executable and I/O file names for each module.

* Submit script commands for executing each module.

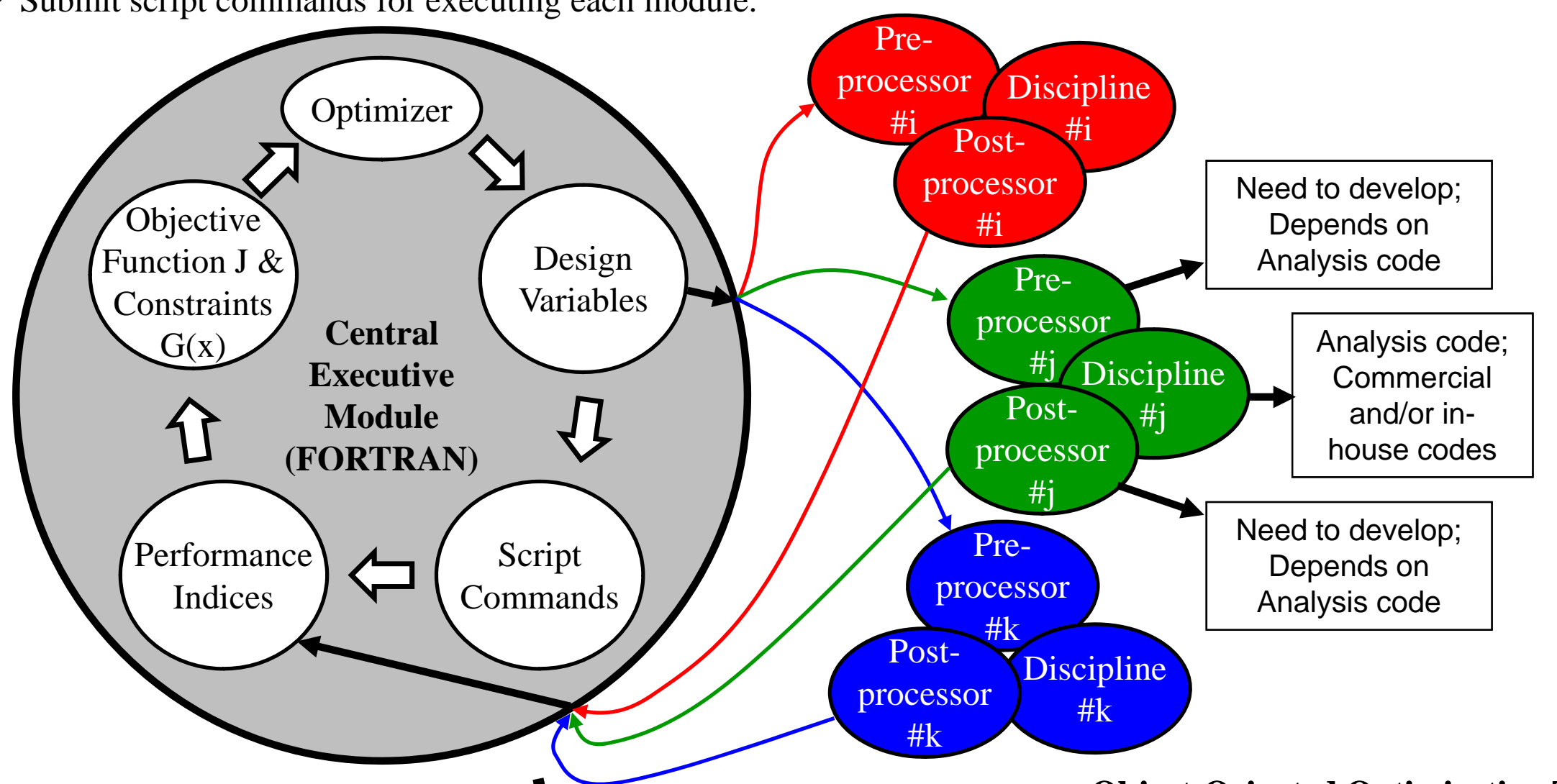

Object-Oriented Optimization Tool 


\section{Introductions (continued)}

Status of the Multi-disciplinary Design, Analysis and Optimization (MDAO) tool

* Structural analyses modules such as computations of the structural weight, stress, deflection, buckling, and flutter and divergence speeds as well as a structural model update module have been developed and incorporated into the $\mathrm{O}^{3}$ tool to build a MDAO tool.

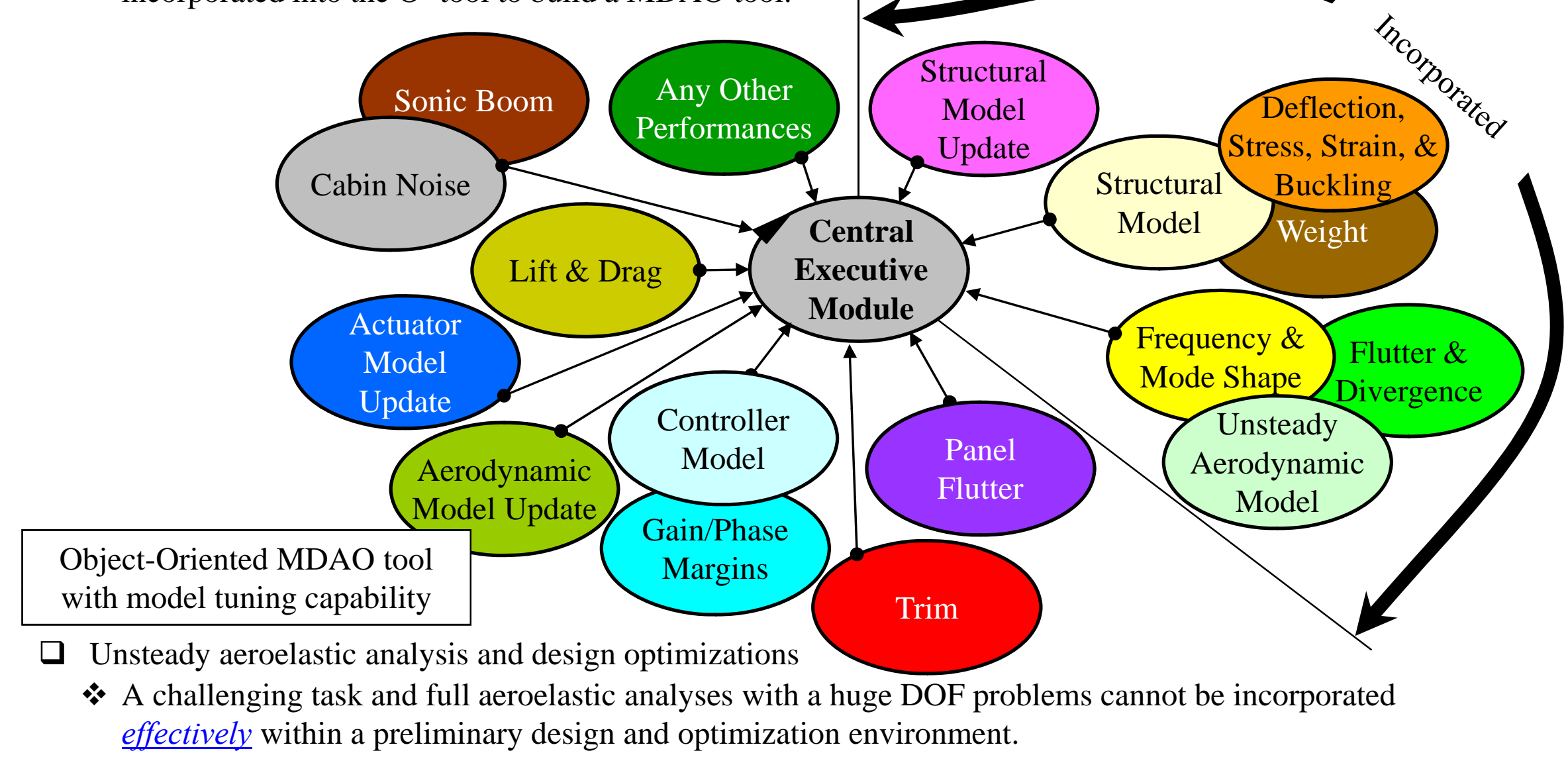

IKHANA (a modified Predator B) aircraft

* With existing AIC data, one Mach number, and 16 reduced frequencies, it takes an average of 30 minutes for a flutter solution on an Intel Core ${ }^{\mathrm{TM}} 22.80 \mathrm{GHz} \mathrm{CPU}$ computer. 


\section{Objectives}

a To be able to perform aeroelastic calculations more quickly and inexpensively by reducing the computation time for generating modal AIC matrices using Basis Function Approximation (BFA) method during the optimization procedure. 


\section{Basis Function Approximation}

The BFA method requires the unsteady aerodynamics to be represented in the frequency domain. The flutter solution can be found by the classic methods, such as rational function approximation, k, p-k, p, root locus et cetera.

- In step 1, define a set of "representative" structural basis function. Intended to capture salient features of the modal responses the airplane to encounter in the various design space (i.e. different mass configurations).

* In this study, mode shapes based on different stiffness and mass configurations of an original eigenvalue problem are used as the basis functions.

* Basis functions used in this study are comparison functions for the original eigenvalue problem.

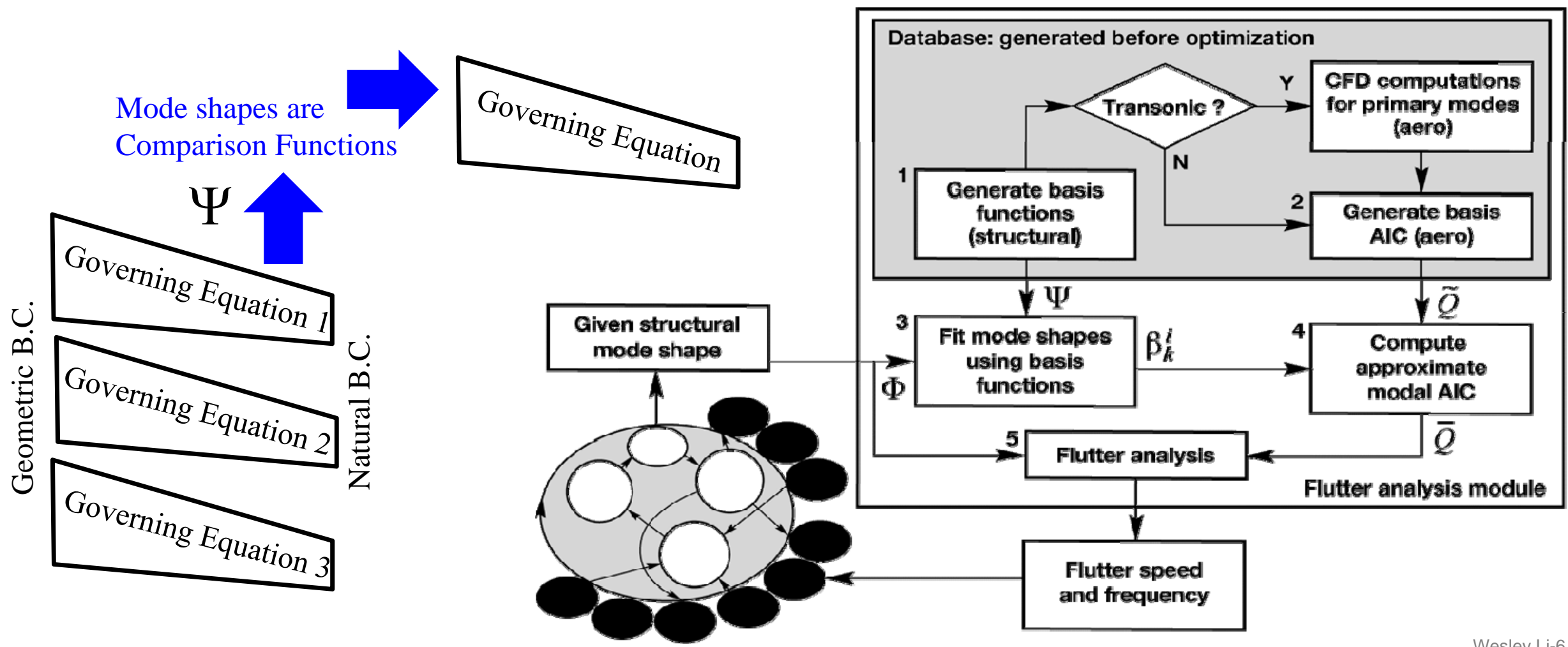




\section{Basis Function Approximation (continued)}

In step 2, compute the "representative" basis AIC matrices corresponding to the representative basis functions defined in step 1 at each Mach number and reduced frequencies. These basis AIC matrices are used as input for approximate modal AIC matrix calculation in step 4.
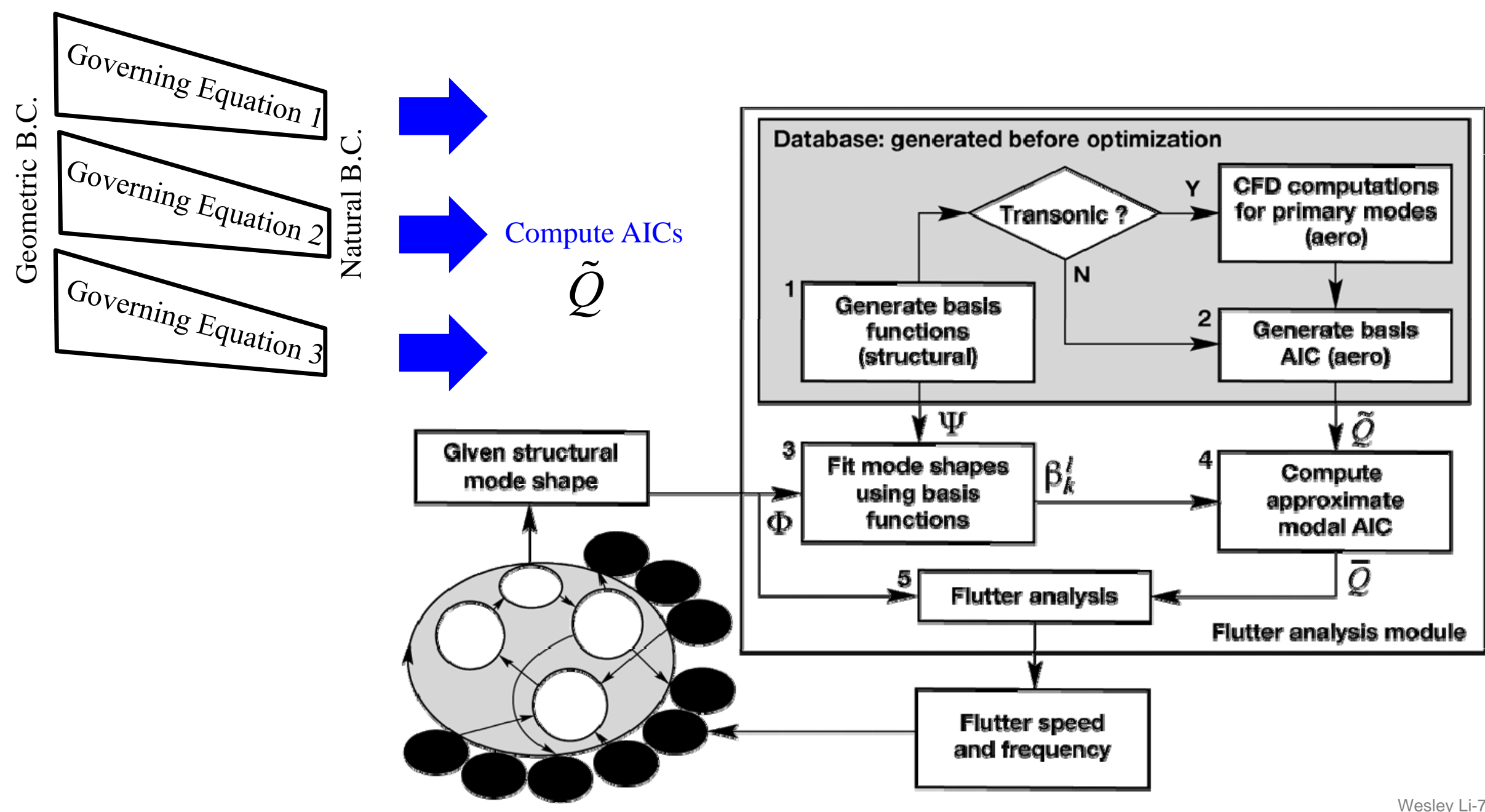


\section{Basis Function Approximation (continued)}

In step 3, decompose each given structural mode shape in a linear combination of the basis functions $\Psi$. The i-th mode shape, $\Phi_{\mathrm{i}}$, is approximated through the use of a least squares fitting together with the following basis functions. $\mathrm{n}$ is number of basis functions.

$$
\phi_{i} \approx \sum_{k=1}^{n} \beta_{k}^{i} \psi_{k}
$$

$\square$ In step 4, compute the approximate modal AIC matrix based on a basis AIC matrix and modal participation factors. This approximate modal AIC matrix will be used for flutter analysis.

$$
\bar{Q}_{i j}=\sum_{r=1}^{n} \sum_{s=1}^{n} \beta_{s}^{i} \beta_{r}^{j} \tilde{Q}_{s r}
$$

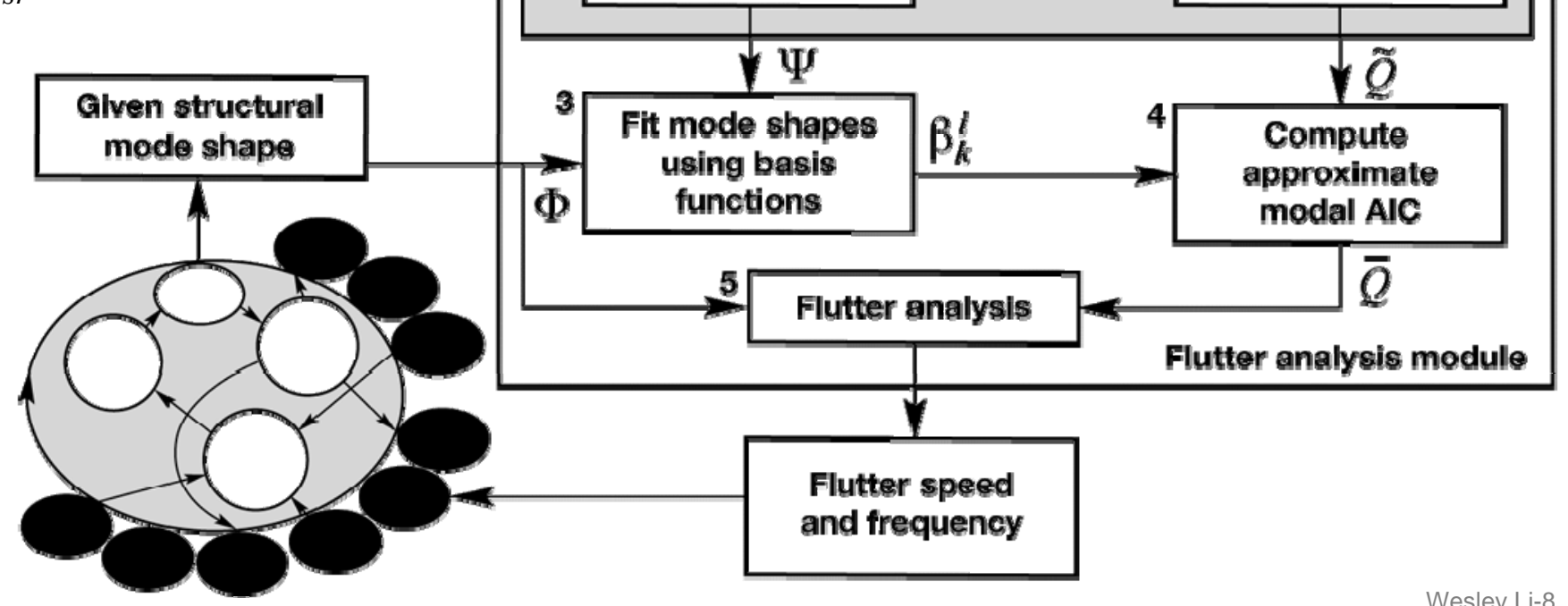




\section{Basis Function Approximation (continued)}

\section{Direct}

Modal Aic $Q_{i j}=\phi_{i}$

General AIC: ndof x ndof

\section{Direct vs. Basis Function Approximation}

\section{BFA}

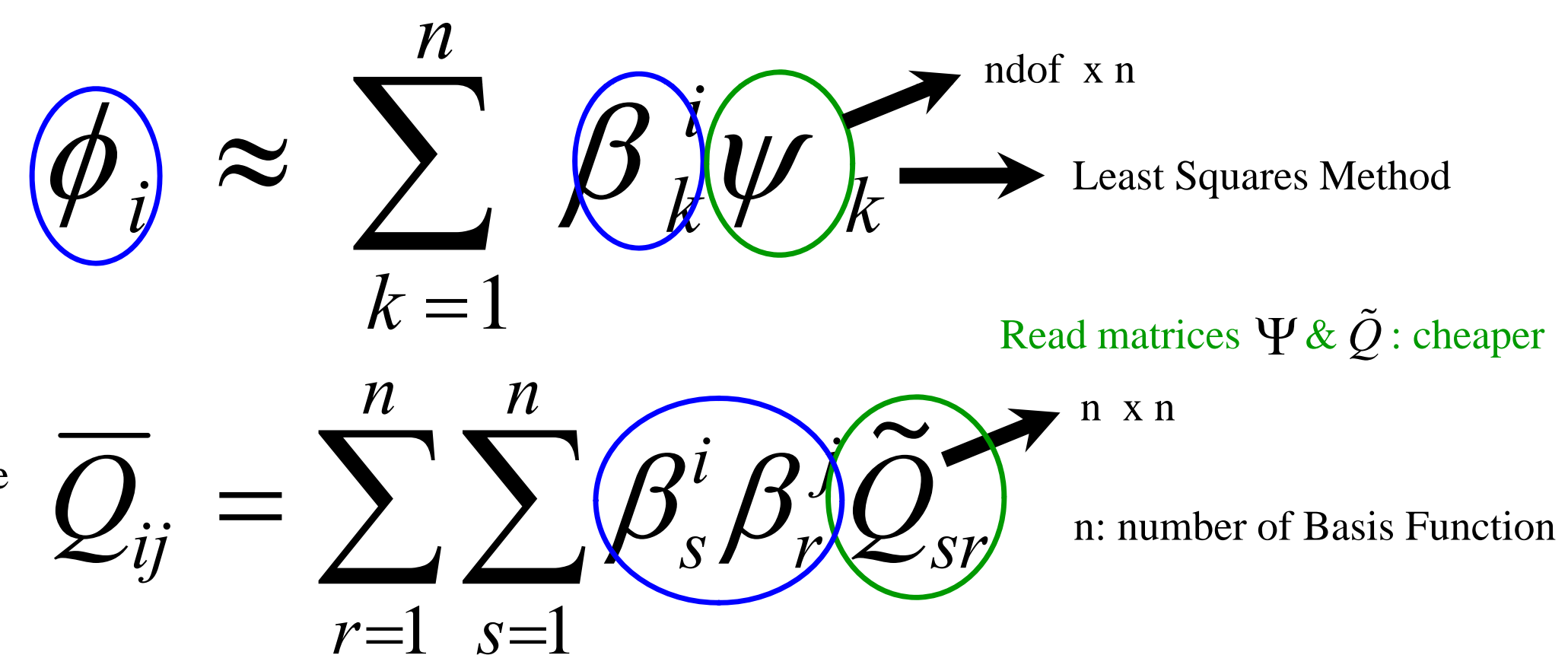




\section{Validations}

In order to validate the proposed BFA method in subsonic flight regimes, the flutter results using this approximate method are compared with the direct flutter results.

* Test case 1 - A modified Aerostructures Test Wing 2 (ATW2)

- Test case 2 - A Hybrid Wing/Body (HWB) aircraft
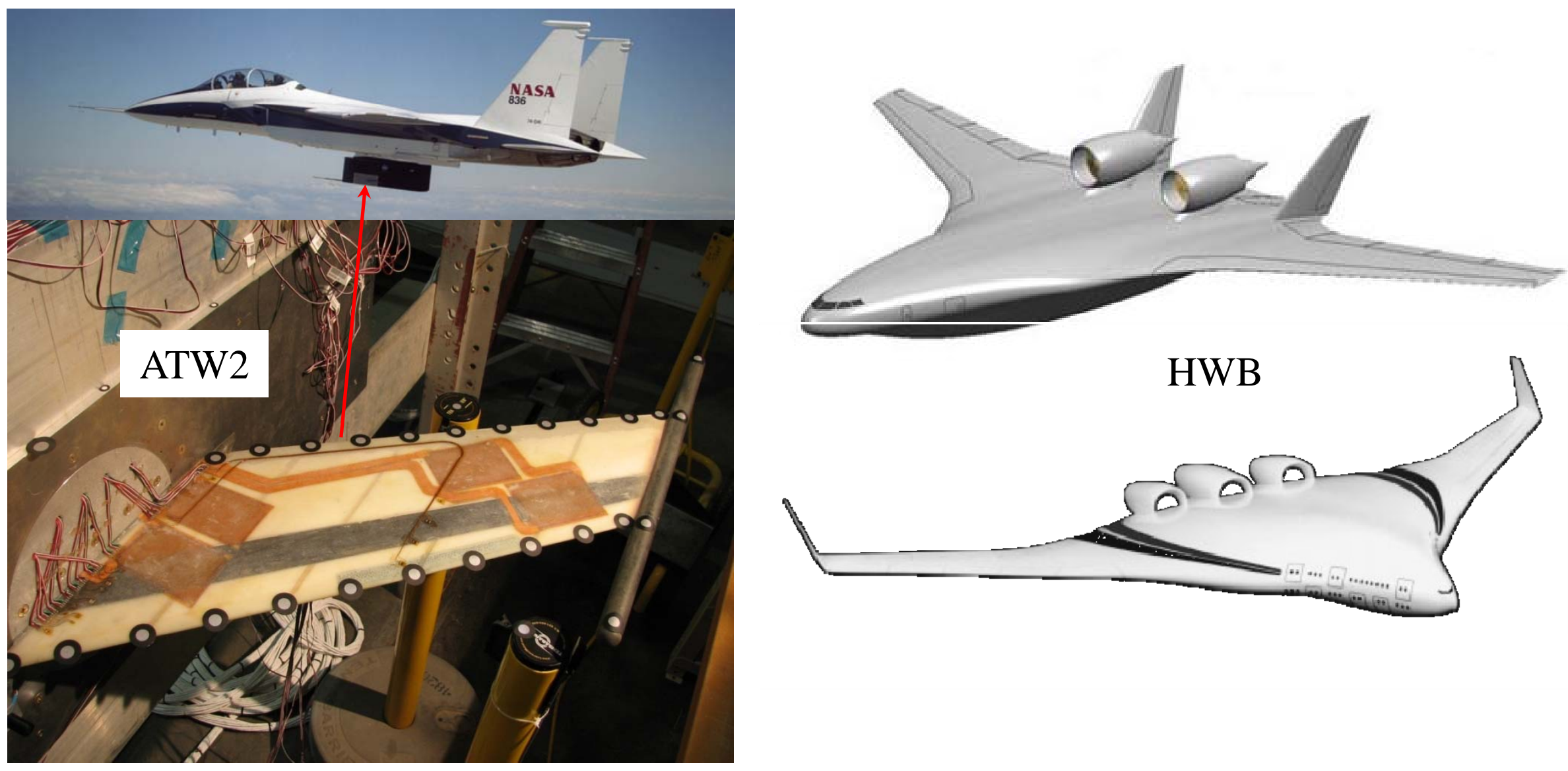


\section{Test case 1: A modified ATW2}

Target configuration

* Mach 0.82, mass=0.0 units, 16 reduced frequencies and 10 structural modes

$\square$ Direct flutter solution

* Using MSC/Nastran and ZAERO

approximation method

* 3 different mass configurations, 0.3, 0.6 , and 0.9 units

* 15 basis functions for each of the configuration, total of 45 basis functions

* Compute the approximate AIC matrix

* Using MSC/Nastran and ZAERO

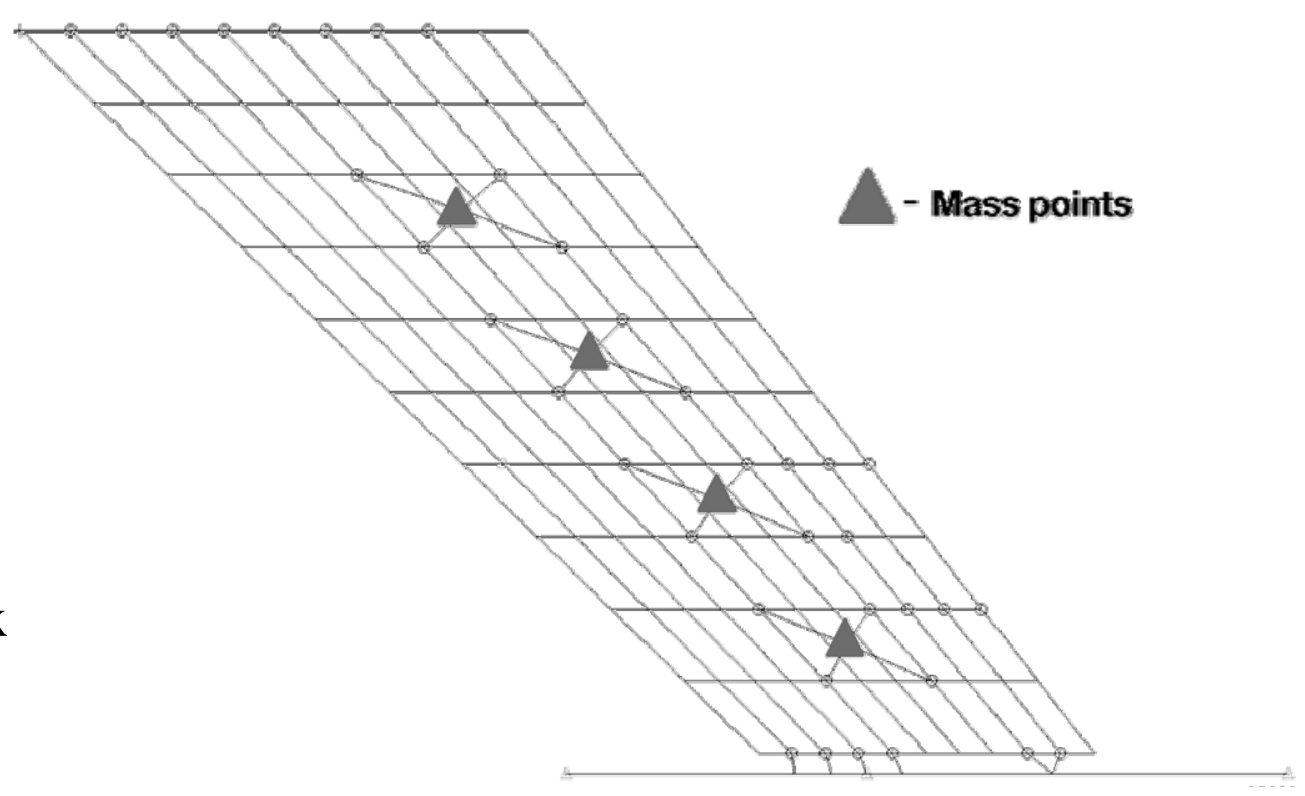




\section{Modified ATW2: Results}

Summary of total weight and center of gravity location for the ATW2 with different fictitious mass configurations

\begin{tabular}{|c|c|c|c|c|}
\hline Mass Configuration & $\mathbf{1}$ & $\mathbf{2}$ & $\mathbf{3}$ & Target \\
\hline Total weight, lb & 4.05 & 5.25 & 6.45 & 2.85 \\
\hline X center of gravity, in & 14.51 & 14.95 & 15.22 & 13.71 \\
\hline Y center of gravity, in & -8.76 & -9.01 & -9.17 & -8.29 \\
\hline Z center of gravity, in & 0.00 & 0.00 & 0.00 & 0.00 \\
\hline Number of modes & 15 & 15 & 15 & 10 \\
\hline
\end{tabular}

Summary of natural frequencies $(\mathrm{Hz})$ of the ATW2 with different fictitious mass configurations

\begin{tabular}{|c|c|c|c|c|}
\hline Mode & Configuration 1 & Configuration 2 & Configuration 3 & Target \\
\hline 1 & 18.10 & 15.98 & 14.45 & 21.32 \\
\hline 2 & 62.81 & 61.91 & 60.60 & 63.55 \\
\hline 3 & 85.87 & 77.10 & 71.84 & 103.18 \\
\hline 4 & 126.60 & 112.29 & 101.86 & 147.79 \\
\hline 5 & 191.68 & 173.89 & 160.47 & 208.55 \\
\hline 6 & 230.10 & 220.25 & 215.18 & 265.53 \\
\hline 7 & 357.83 & 317.80 & 290.89 & 408.75 \\
\hline 8 & 403.33 & 383.13 & 367.52 & 434.86 \\
\hline 9 & 450.24 & 444.21 & 426.69 & 472.85 \\
\hline 10 & 532.04 & 477.94 & 448.37 & 589.94 \\
\hline 11 & 628.72 & 616.37 & 600.44 & \\
\hline 12 & 633.07 & 630.08 & 628.51 & \\
\hline 13 & 708.57 & 707.15 & 684.02 & \\
\hline 14 & 729.18 & 726.37 & 705.74 & \\
\hline 15 & 782.46 & 728.68 & 725.76 & \\
\hline
\end{tabular}




\section{Modified ATW2: Mode Shapes}

Structural finite element model mode shapes and frequencies of the ATW2 with target mass configuration (direct method).

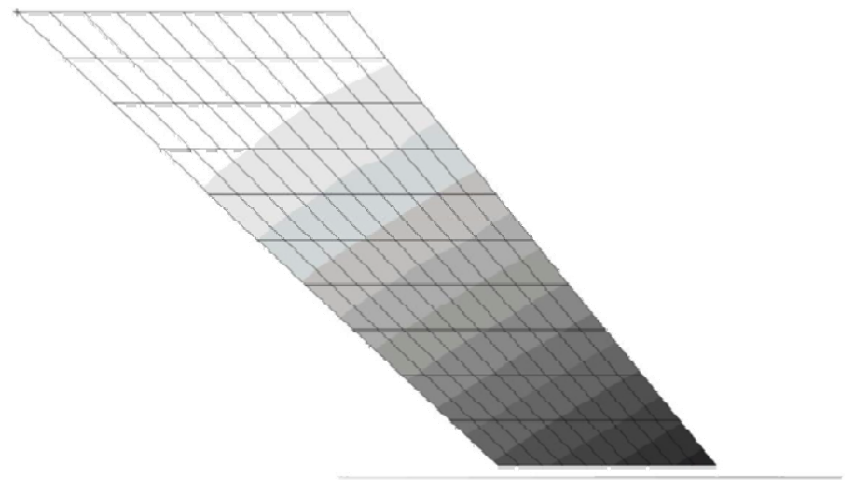

Mode 1 (First bending), $21.3 \mathrm{~Hz}$

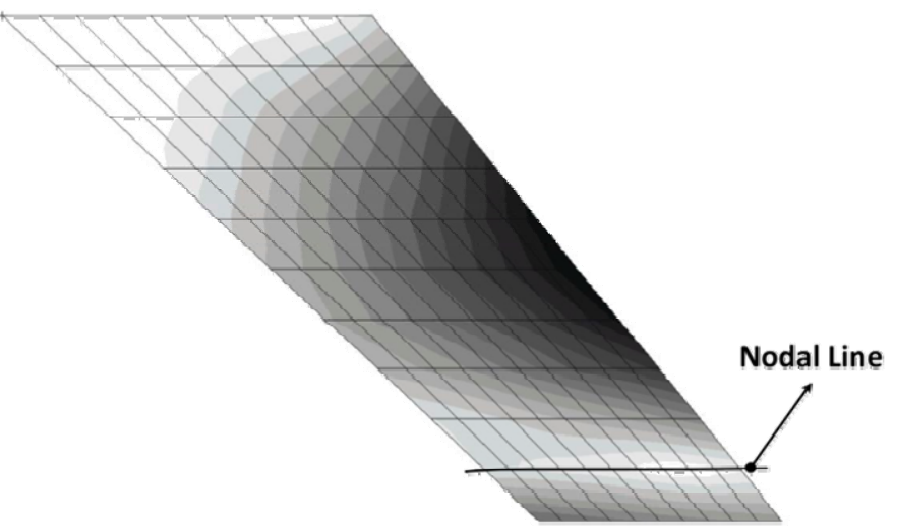

Mode 3 (Second bending and first torsion), $103.2 \mathrm{~Hz}$.

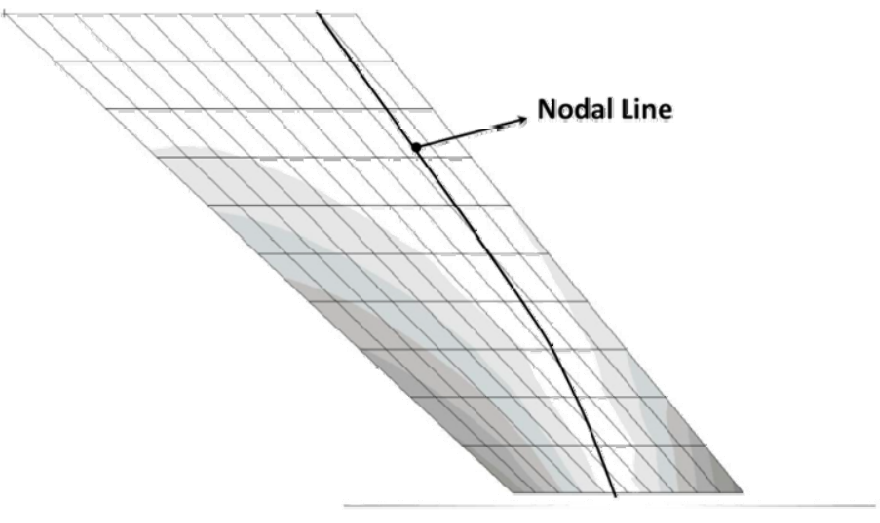

Mode 2 (Second bending and first torsion), $63.6 \mathrm{~Hz}$. 


\section{Modified ATW2: Mode Shapes Comparison}

$\square$ Structural finite element model mode shapes error of the ATW2 with target mass configuration.

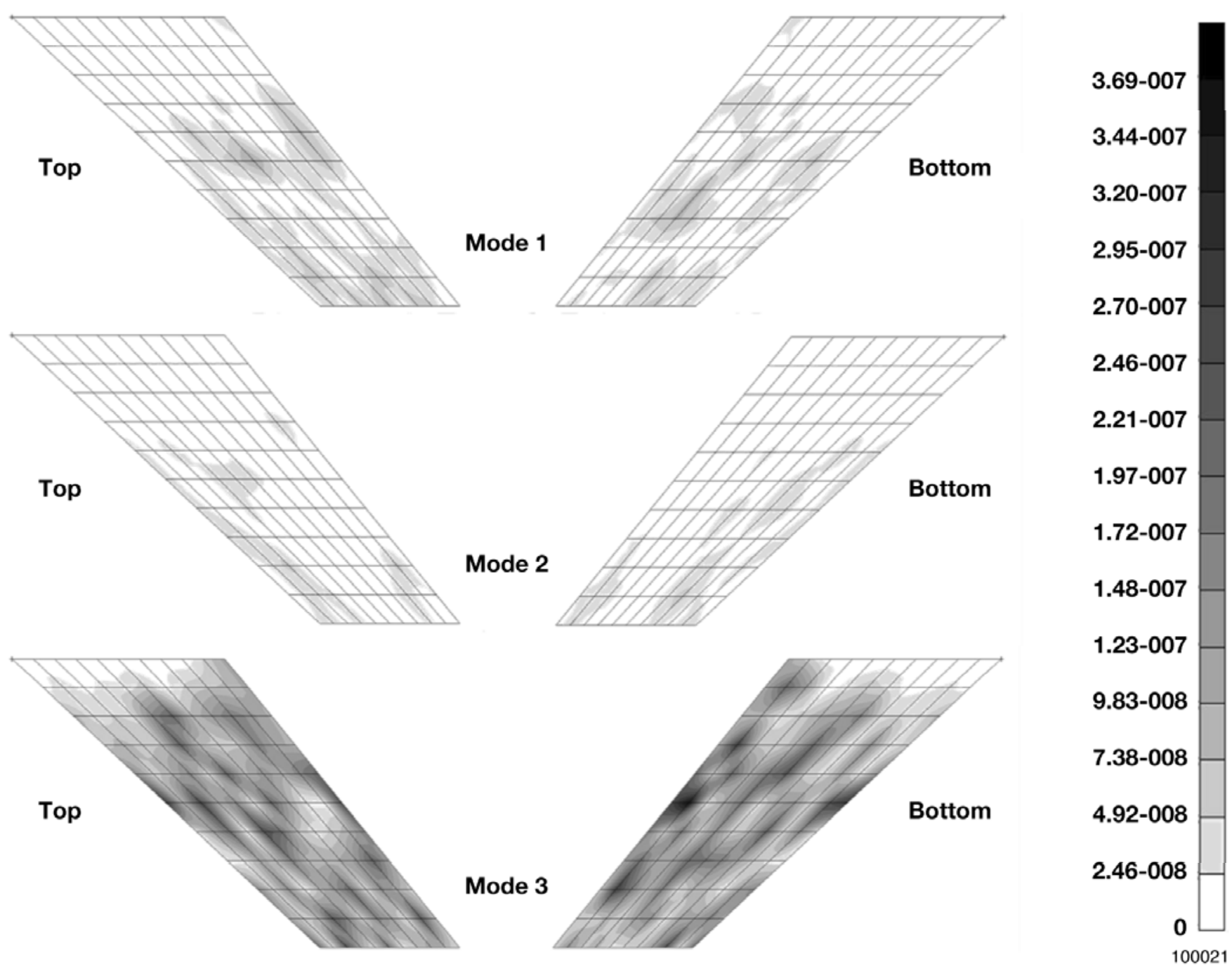




\section{Modified ATW2 Flutter Results - Vg Plots}

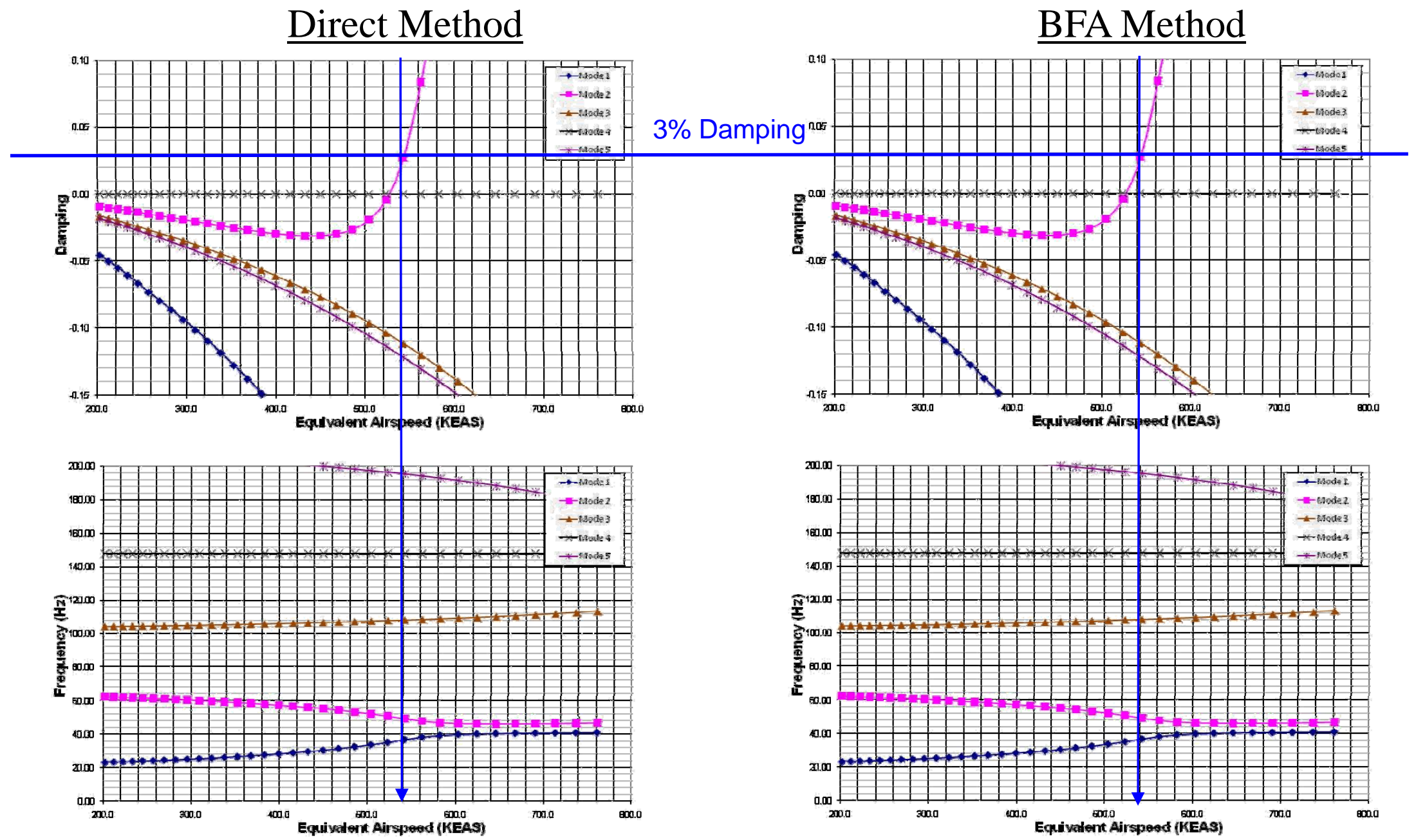

\begin{tabular}{|c|c|c|c|}
\hline & BFA Method & Direct Method & Error (\%) \\
\hline Flutter Speed (keas) & 543.59 & 543.59 & 0.0 \\
\hline Flutter Frequency (Hz) & 49.01 & 49.01 & 0.0 \\
\hline
\end{tabular}




\section{Test case 2: A HWB Aircraft}

- Test configuration

* Mach 0.5, mass=0.0 units, 14 reduced frequencies and 40 structural modes

$\square$ Direct flutter solution

* Using MSC/Nastran and ZAERO

Approximation method

* 3 different fuel weight configurations

* 50 basis functions for each of the configuration, total of 150 basis functions

* Compute the approximate AIC matrix Using MSC/Nastran and ZAERO

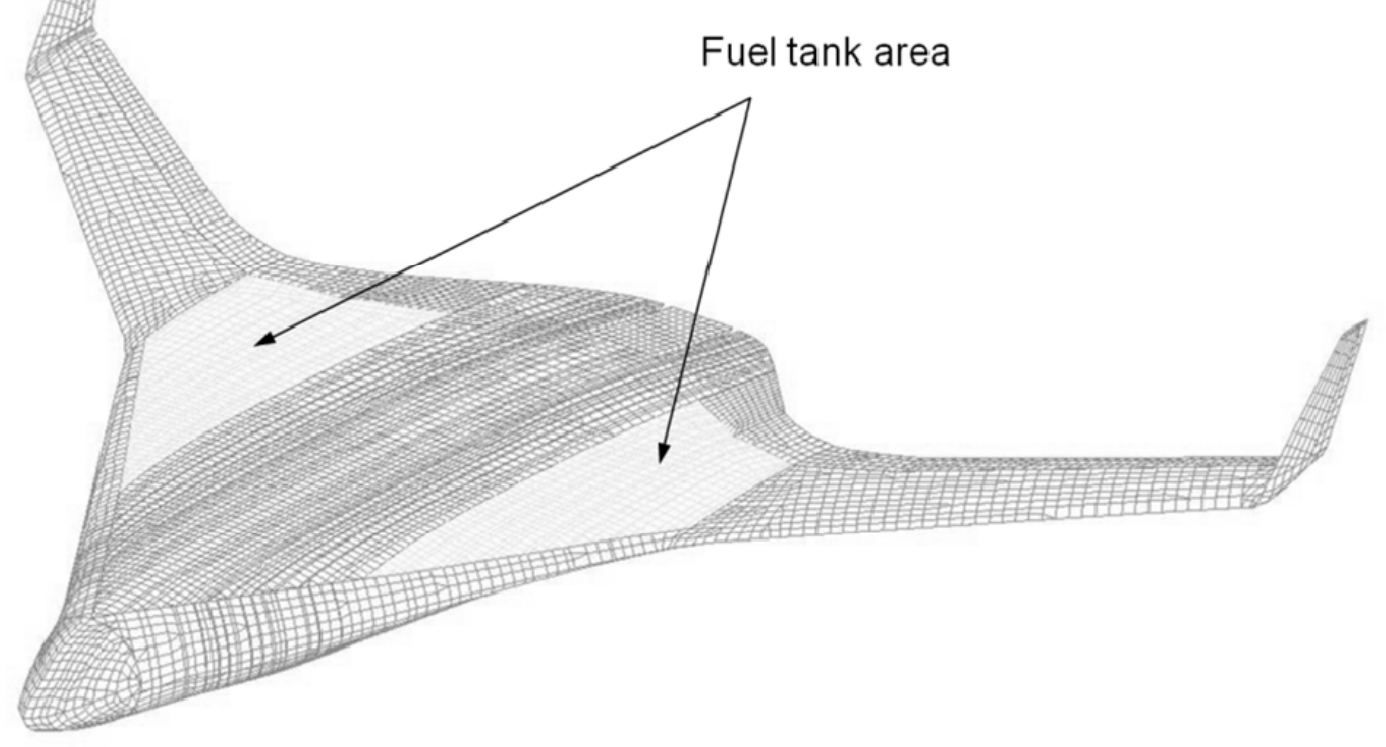




\section{HWB: Mode Shapes}
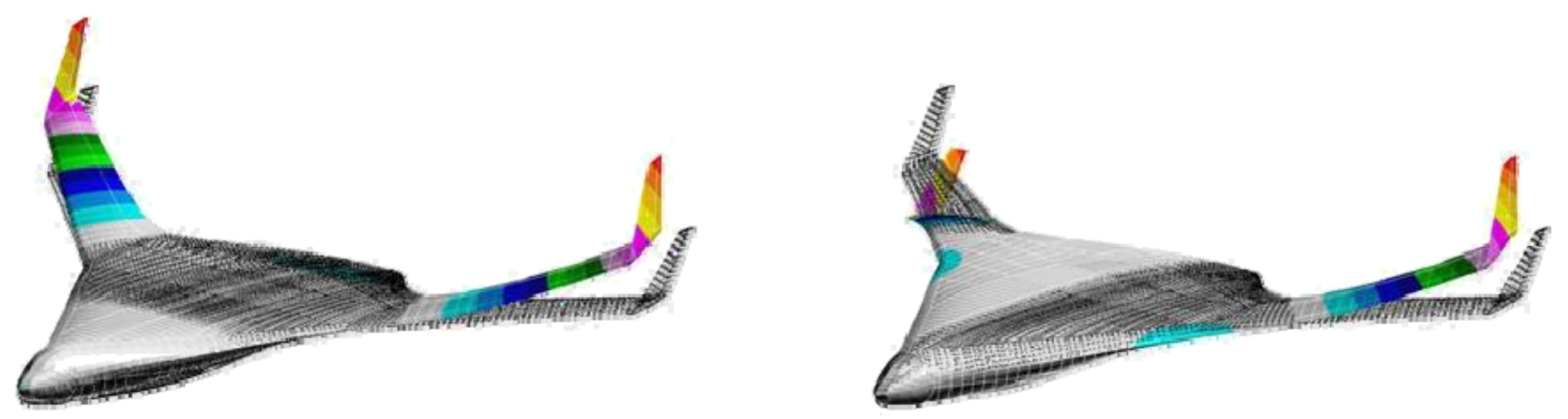

Flexible Mode 1 (1 $1^{\text {st }}$ sym bending)

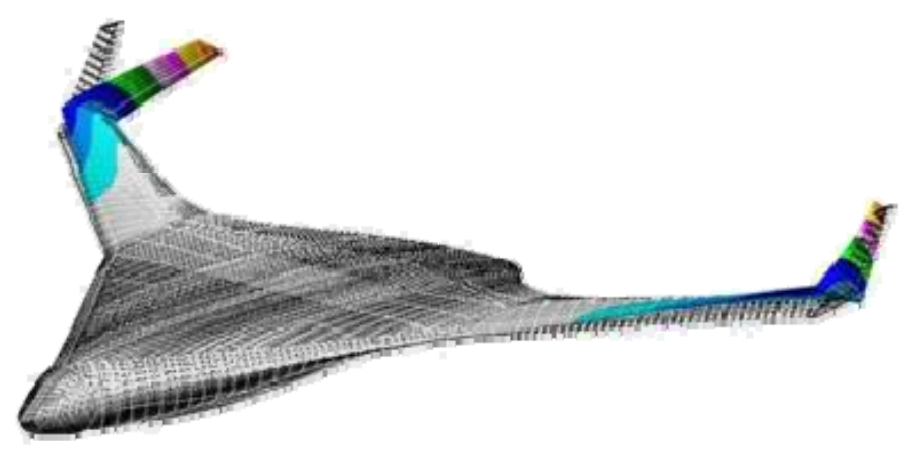

Flexible Mode 3 (1 $1^{\text {th }}$ sym torsion)
Flexible Mode 2 (1 $1^{\text {ft }}$ enti-sym bending)

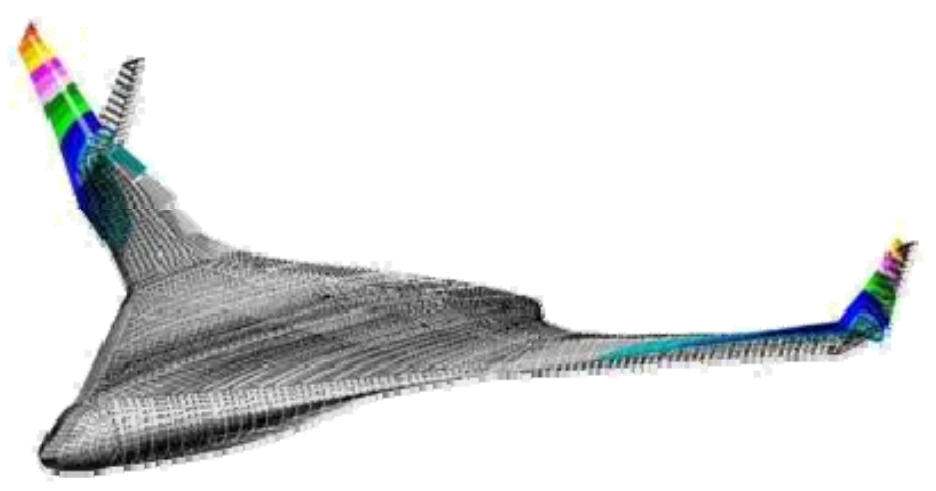

Flexible Mode 4 (1 $1^{\text {ft }}$ anti-sym torsion) 


\section{HWB: Results}

- Summary of total weight and center of gravity location for the HWB with different fuel weight configurations

\begin{tabular}{|c|c|c|c|c|}
\hline Mass Configuration & $\mathbf{1}$ & $\mathbf{2}$ & $\mathbf{3}$ & Target \\
\hline Total Weight, lb & 403781 & 478941 & 591681 & 535311 \\
\hline X center of gravity, in & 1337.60 & 1334.51 & 1331.34 & 1332.76 \\
\hline Y center of gravity, in & 0.75 & 0.46 & 0.16 & 0.29 \\
\hline Z center of gravity, in & 20.89 & 21.35 & 21.82 & 21.61 \\
\hline Number of modes & 50 & 50 & 50 & 40 \\
\hline
\end{tabular}

Summary of natural frequencies $(\mathrm{Hz})$ of the HWB with different fuel weight configurations

\begin{tabular}{|c|c|c|c|c|}
\hline Mode & Configuration 1 & Configuration 2 & Configuration 3 & Target \\
\hline 1 & 1.60 & 1.54 & 1.47 & 1.50 \\
\hline 2 & 1.74 & 1.64 & 1.53 & 1.58 \\
\hline 3 & 3.52 & 3.35 & 3.13 & 3.24 \\
\hline 4 & 3.64 & 3.45 & 3.22 & 3.33 \\
\hline 5 & 5.24 & 5.00 & 4.54 & 4.83 \\
\hline 6 & 5.26 & 5.04 & 4.54 & 4.89 \\
\hline 7 & 6.42 & 5.42 & 4.68 & 4.92 \\
\hline 8 & 6.42 & 5.43 & 4.76 & 4.92 \\
\hline 9 & 6.92 & 6.66 & 5.81 & 6.31 \\
\hline 10 & 7.12 & 6.68 & 5.82 & 6.31 \\
\hline 11 & 7.33 & 6.95 & 6.11 & 6.39 \\
\hline 12 & 7.65 & 6.95 & 6.32 & 6.48 \\
\hline 13 & 8.22 & 7.03 & 6.69 & 6.85 \\
\hline 14 & 8.23 & 7.40 & 7.06 & 7.22 \\
\hline
\end{tabular}




\section{HWB Flutter Results - Vg Plots}

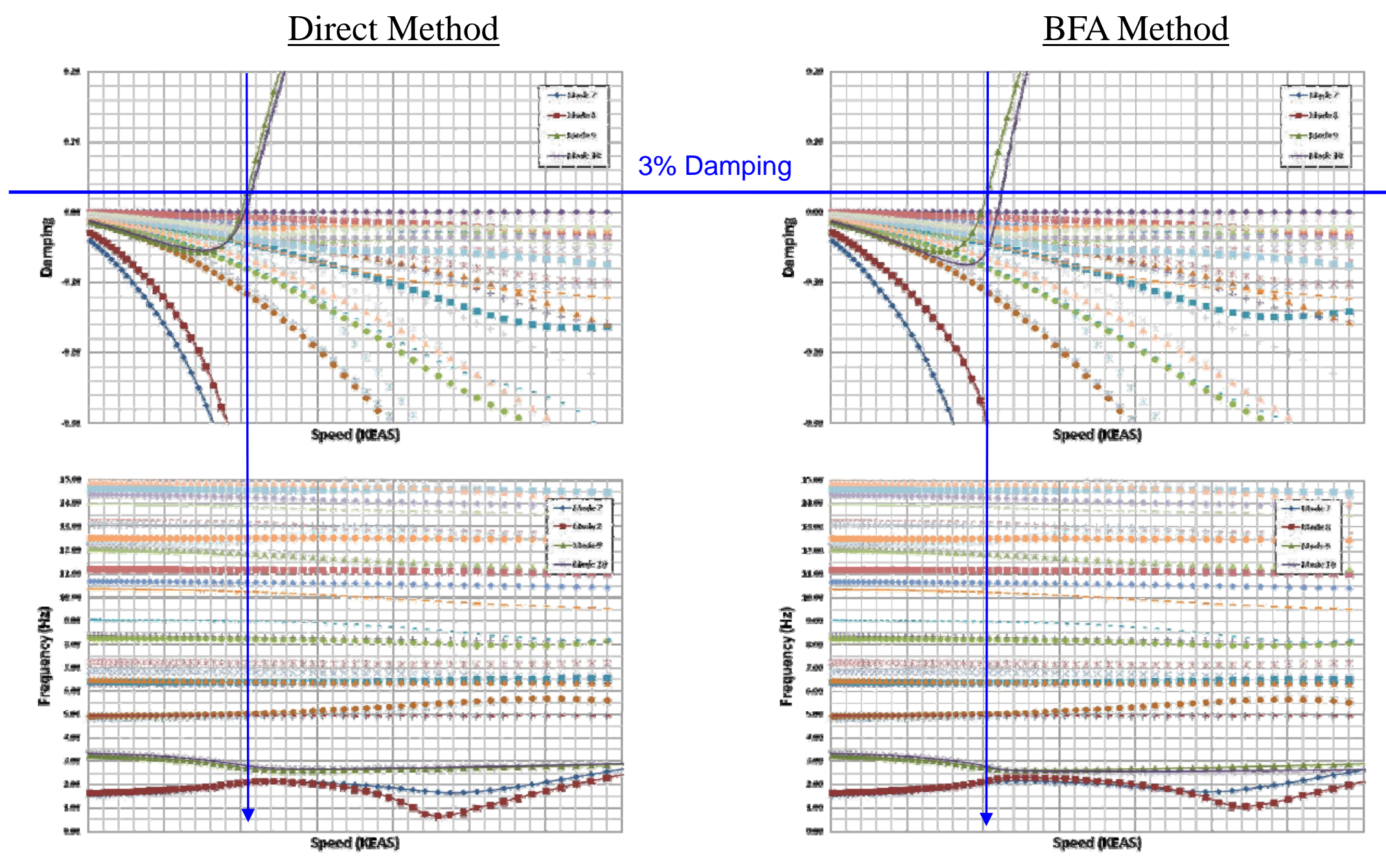

\begin{tabular}{|c|c|c|c|}
\hline & BFA Method & Direct Method & Error (\%) \\
\hline Flutter Speed (normalized) & 0.991 & 1.0 & 0.86 \\
\hline Flutter Frequency (Hz) & 2.671 & 2.667 & 0.15 \\
\hline
\end{tabular}




\section{Computational Cost}

C Computational cost comparison of BFA and Direct method

\begin{tabular}{|c|c|c|}
\hline Test Case & ATW2 & HWB \\
\hline Number of nodes & 269 & $\sim 24000$ \\
\hline Number of structural modes & 10 & 40 \\
\hline Number of reduced frequencies & 16 & 14 \\
\hline Direct method elapsed time & $43 \mathrm{~s}$ & $50 \mathrm{~min}$ \\
\hline BFA method elapsed time & $8 \mathrm{~s}$ & $9 \mathrm{~min}$ \\
\hline
\end{tabular}

BFA is 5X faster than Direct. 


\section{Conclusions}

A technique for approximating the modal Aerodynamic Influence Coefficient (AIC) matrix by using basis functions has been developed and validated, and a process for using the resulting AIC matrix in aeroelastic analysis and design optimization has been proposed.

The approximation method has been applied to the aeroelastic analyses, and the results are essentially identical to those using direct solution. The technique presented has been shown to offer consistent flutter speed prediction on an ATW2 configuration and a HWB type vehicle with negligibly small loss in precision.

These results may have practical significance in the analysis of aircraft aeroelastic calculation and application in parametric flutter analyses as well as more efficient multidisciplinary design and optimization studies.

This method computes AICs that are functions of the changing parameters being studied and are generated within minutes of CPU time instead of hours. The improvement could lead to a more efficient design optimization cycle.

The Basis Function Approximation yields significant improvements in computational efficiency as compared to the original approach, thereby meeting the objective of this study. 


\section{Questions?}

